\title{
LA PARTICIPACIÓN FEMENINA EN PUBLICACIONES COLOMBIANAS DE ECONOMÍA Y ADMINISTRACIÓN INDEXADAS EN SCOPUS (1974-JUNIO DE 2014)*
}

\author{
JENNY-PAOLA LIS-GUTIÉRREZ"** \& CLORITH-ANGÉLICA BAHOS-OLIVERA*** \\ FUNDACIÓN UNIVERSITARIA KONRAD LORENZ - UNIVERSIDAD NACIONAL DE COLOMBIA
}

Recibido/ Received/ Recebido: 05/02/2015 - Aceptado/ Accepted/ Aprovado: 07/04/2016

\section{Resumen}

A partir de la construcción de: (i) indicadores de género (distribución horizontal, distribución vertical, índice de feminidad, índice de masculinidad, índice de Duncan, índice de segregación, índice de contribución al sexismo e índice de interacción), (ii) un análisis bibliométrico descriptivo y evaluativo, segmentado por el sexo de los autores, y (iii) representaciones de cartografía temática y estadística, se pretendió establecer si existe una brecha de género en la producción académica nacional en Economía y Administración, esto, a pesar de que el número de autoras en ciencias económicas se haya incrementado en años recientes. Los resultados indican que los artículos con mujeres como autoras principales no superaron el $27 \%$ y como co-autoras solo fue del $24 \%$. A su vez se identificó que los mejores indicadores en cuanto a reiteración de las citaciones (índices $\mathrm{H}, \mathrm{R}$ y A) los obtienen los artículos escritos por hombres sin co-autoría femenina. Finalmente se logró establecer que los indicadores de género calculados con respecto al número de autores, reiteran la brecha de género y la masculinización en la publicación científica en ciencias económicas, sin embargo, no hay evidencia de segregación en las tres áreas analizadas.

Palabras clave: Bibliometría; Cienciometría; Economía; Administración; Índices bibliométricos; Indicadores de género; Brecha de género; Scopus.

\section{WOMEN'S PARTICIPATION IN ECONOMY AND ADMINISTRATION COLOMBIAN PUBLICATIONS INDEXED IN SCOPUS (1974 - JUNE 2014)}

\begin{abstract}
From the construction of: (i) gender indicators (horizontal distribution, vertical distribution, rate of femininity, masculinity index, Duncan index, segregation index, contribution to sexism index and interaction index), (ii) a descriptive and evaluative bibliometric analysis, segmented by sex of the authors, and (iii) representations of thematic and statistical cartography, was intended to establish whether there is a gender gap in national academic production in Economics and Management, this, despite the number of female authors in economics has increased in recent years. The results

Candidata a doctora en Ingeniería, en el área de Industria y Organizaciones (Universidad Nacional de Colombia), Magíster en Análisis de problemas políticos, económicos e internacionales (Universidad Externado de Colombia), Magíster en Sociedades contemporáneas comparadas Europa-América Latina (Paris III- Sorbonne Nouvelle), especialidad en Geografía y Planeación (IHEAL), Economista (Universidad Nacional de Colombia). Se desempeña actualmente como Directora del Centro de Investigaciones de la Escuela de Negocios de la Fundación Konrad Lorenz, y docente catedrática de las universidades Externado de Colombia, Central y del Instituto de Altos Estudios para el Desarrollo. Dirección postal: Carrera 9 bis No. 62 - 43 (Bogotá - Colombia). Teléfono: (057-1) 3472311 Ext. 228. Correo electrónico: jenny.lis@konradlorenz.edu.co

..." Estudiante de la Maestría en Estudios Sociales de la Ciencia y Economista (Universidad Nacional de Colombia). Profesional de Centro de Apoyo a la Investigación Económica-CAIE del Banco de la República. Correo electrónico: thelik313@gmail.com
\end{abstract}

Artículo de reflexión 
indicate that articles with women as main authors did not exceed $27 \%$ and as co-authors was only 24\%. In turn it was identified that the best indicators as to repeated citations (index H, R and A) get articles written by men without female co-authorship. Finally it was established that gender indicators calculated relative to the number of authors reiterate the gender gap and masculinization in scientific publication in economics, however, there is no evidence of segregation in the three areas analyzed. Keywords: Bibliometrics; Scientometrics; Economy; Management; Bibliometric index; Gender indicators; Gender gap; Scopus.

\section{A PARTICIPAÇÃO FEMININA EM PUBLICAÇÕES COLOMBIANAS DE ECONOMIA E ADMINISTRAÇÃO INDEXADAS EM SCOPUS (1974 - JUNHO DE 2014) \\ Resumo}

A partir da construção de: (i) indicadores de gênero (distribuição horizontal, distribuição vertical, índice de feminidade, índice de masculinidade, índice de Duncan, índice de segregação, índice de contribuição ao sexismo e índice de interação), (ii) uma análise bibliométrica descritiva e avaliativa, segmentada pelo sexo dos autores, e (iii) representações de cartografia temática e estatística, pretendeu-se estabelecer se existe uma brecha de gênero na produção acadêmica nacional em economia e administração, apesar de que o número de autoras em ciências econômicas tenha se incrementado nos anos recentes. Os resultados indicam que os artigos com mulheres como autoras principais não superaram $27 \%$ e como coautoras só foi de $24 \%$. Por outro lado identificou-se que os melhores indicadores quanto a reiteração das citações (índices $\mathrm{H}, \mathrm{R}$ e A) são obtidos por artigos escritos por homens sem coautoria feminina. Finalmente conseguiu-se estabelecer que os indicadores de gênero calculados com relação ao número de autores, reiteram a brecha de gênero e a masculinização na publicação científica em ciências econômicas, não havendo evidência, no entanto, de segregação nas três áreas analisadas.

Palavras chave: Bibliometria; Cienciometria; Economia; Administração; Índices bibliométricos; Indicadores de gênero; Brecha de gênero; Scopus.

Lis-Gutiérrez, J-P. \& Bahos-Olivera, C-A. (2016). La participación femenina en publicaciones colombianas de economía y administración indexadas en Scopus (1974 - junio de 2014). En: Revista de la Facultad de Ciencias Económica: Investigación y Reflexión. rev.fac.cienc.econ, XXIV (2), DOI: http://dx.doi.org/10.18359/rfce.2219

JEL: 010, R19, Y90, Z00.

\section{Introducción}

El interés por la comunicación científica y la bibliometría ha ido en aumento en décadas recientes. Al igual que los análisis de género aplicados a diferentes disciplinas. A pesar de lo anterior, son pocos los trabajos que relacionan estas dos aproximaciones.

En este documento se propone un análisis sobre la participación de las mujeres en las publicaciones de origen colombiano en economía y administración, basado en la información del servicio de indización y resumen SCOPUS ${ }^{1}$ durante el período enero de

Sistema de información científica desarrollado por Elsevier en noviembre de 2004, la información puede analizarse considerando 31 campos de conocimiento, 313 categorías temáticas (de acuerdo a la clasificación de Scopus), 10 regiones geográficas, 233 países, 21.500 revistas arbitradas, de las cuales más de 4.200 son de libre acceso, 113.000 libros, 60 millones de registros en total (Scopus, 2016). 
1974 y junio de 2014. Esto con el fin de identificar si existe una brecha de género en la producción académica que se visibiliza en uno de los índices bibliográficos más reconocidos.

Los indicadores que serán considerados en este trabajo dan cuenta tanto de la bibliometría descriptiva como evaluativa ${ }^{2}$ : volumen de la producción medido mediante el número de documentos, tipología de documentos, idiomas de escritura, número de citaciones, filiación institucional de los autores, fuentes, principales revistas (González, Guerrero \& Moya, 2009), análisis de países, los índices H, G, R, i10 y A (Hirsch, 2005; Egghe, 2006; Jin, Liang, Rousseau \& Egghe, 2007; Túñez \& de Pablos, 2013) $e$ indicadores de género distribución horizontal, distribución vertical, índice de feminidad, índice de masculinidad, índice de Duncan, índice de segregación, índice de contribución al sexismo e índice de interacción. Para algunos de los indicadores se empleará la representación de cartografía estadística ${ }^{3}$.

La estructura del documento es la siguiente. Inicialmente se hará una revisión de la literatura reciente. En la segunda parte se realiza una descripción de los indicadores básicos de las publicaciones. Posteriormente, se elabora un análisis de las citaciones recibidas por los documentos y los índices $\mathrm{H}, \mathrm{G}, \mathrm{R}$, i10 y A. En el siguiente segmento se presentan los resultados de los indicadores de género. En la última

2 Los estudios bibliométricos se clasifican dependiendo de las fuentes y los análisis que se realizan. En el primer caso, pueden estar basados en: bibliografías; servicios de indización y resúmenes (generalmente se emplean los Índices bibliográficos de citaciones): referencias o citaciones (análisis de citaciones); y en directorios o catálogos colectivos de títulos de revistas (Araújo \& Arencibia, 2002). En el segundo caso, existen dos subcategorías: la bibliometría "descriptiva, que trata de aspectos puramente cuantitativos, como distribución geográfica, documental, temática y su productividad y [la] evaluativa, que añade a la primera estudios de evaluación de la actividad científica" (Rubio, 1999, p. 1).

3 La importancia de usar este tipo de herramientas, de acuerdo con Jatobá \& Gomes (2012, p. 63), reside en que: "O uso da cartografia digital para o ensino da Geocartografia é importante por se tornar um instrumento capaz de envolver as pessoas para análise de sua própria realidade e visualizar informações complexas com maior agilidade e qualidade, sem esquecer que a atividade se torna mais prazerosa e menos cansativa". parte, se expondrán algunas consideraciones finales a manera de conclusiones.

\section{Revisión de la literatura}

\subsection{Los estudios bibliométricos recientes}

Pritchard (1969, p. 348) definió la bibliometría como "la aplicación de los métodos estadísticos y matemáticos dispuestos, para definir los procesos de la comunicación escrita, la naturaleza y el desarrollo de las disciplinas científicas mediante técnicas de recuento y análisis de la comunicación” Rehn \& Kronman (2008) por su parte, la entendieron como una herramienta de comparación de las publicaciones en investigación, en un tema en específico, asociado a su evolución e impacto generado, a partir de indicadores. Estos últimos corresponden a datos estadísticos que permiten analizar las distintas características de las publicaciones científicas, con base en la difusión y transmisión del conocimiento derivado de la investigación (Filippo \& Fernández, 2002).

En este apartado se realizará una revisión de los estudios recientemente en los cuales se aplican herramientas bibliométricas. En los análisis de campos de investigación específicos se encuentran los trabajos de Costa (2015) quien analiza la innovación en la enseñanza en finanzas entre 2005 y 2015. Por su parte, Arias, Velasco \& Novo (2015) estudiaron los fundamentos y las nuevas tendencias de la violencia de género y Restrepo \& Urbizagástegui (2016) analizaron las características de las publicaciones en ISI y Scopus sobre pueblos indígenas. El documento de Martínez et al. (2014), identificó mediante cocitaciones la base intelectual de la fisioterapia. Por su parte, López et al. (2014a), estudió la producción española sobre atención primaria, entre 2008 y 2012; Armfield et al. (2014) realizó un análisis de 17.932 publicaciones para el campo de la telemedicina; Portugal et al. (2014), consideraron el período 1980-2010 sobre la literatura de negocios internacionales en fusiones y adquisiciones.

En este mismo ámbito se encuentran el artículo de Yu, Davis \& Dijkeman (2014) discutieron la evolución del campo de la investigación de la simbiosis 
industrial (IS), en relación con la ecología industrial (IE), mediante los análisis de citas, cocitación y redes, identificaron el desarrollo de temas de investigación y la evolución de la red de investigación. Igualmente, Brones, Monteiro de Carvalho \& de Senzi Zancul (2014) buscaron establecer la intersección entre el ecodiseño y la gestión de proyectos, sin embargo, el análisis de la literatura reveló que los principios de gestión de proyectos se abordan de manera limitada en los artículos especializados sobre diseño ecológico, y que no hay artículos específicos sobre la gestión del proyecto que consideren las cuestiones de dirección de desarrollo de productos del medio ambiente y la sostenibilidad ambiental.

Otro ámbito de reciente aplicación de bibliometría está asociado con el análisis de métricas para los autores y el perfeccionamiento de las técnicas bibliométricas. Tales son los casos de los trabajos de Machado et al. (2016) quienes propusieron la aplicación de las leyes de la bibliometría para identificar patrones en las estructuras de contratación de docentes de las instituciones de educación superior. Delgado (2016) quien a partir de Google Scholar propuso nuevas técnicas. Por su parte, Ortiz \& Hidalgo (2016) aplicaron a la co-autoría la teoría de grafos para identificar comunidades académicas. Arias (2015) presentó nuevas aproximaciones metodológicas a partir de la estadística multivariante, de los métodos Biplot y análisis de redes sociales, para estudiar los patrones de colaboración.

Igualmente, Ale Ebrahim et al. (2014) estudiaron el caso de dos investigadores que utilizan herramientas de marketing para sus publicaciones, encontrando evidencia de que la visibilidad artículo mejora en gran medida el impacto, medido por la citación. A su vez, el trabajo de Abramo et al. (2014) analizó en qué medida los científicos italianos más productivos logran artículos muy citados y qué porcentaje de los artículos más citados se logra por los científicos que son los más productivos en Web of Science. Brzezinski (2014), evaluó en la distribución de las citas la "ley de potencia" (power-law behaviour) y encontró que su aplicación es adecuada para los trabajos más citados en Física y Astronomía, pero no para el resto de áreas. Vieira, Cabral \& Gomes (2014) analizaron el poder predictivo de los indica- dores bibliométricos frente a los resultados de las evaluaciones por pares y la obtención de los niveles académicos de profesor Asociado y Catedrático en universidades portuguesas.

Una tercera manera de clasificar los trabajos recientes son aquellos que critican las métricas actuales. Por ejemplo, el documento de Bouyssou \& Marchant (2014) realizó una revisión, comparación y crítica de los índices y ranking que permiten evaluar los autores. Amodio \& Brugnano (2014) mostraron las debilidades de las métricas para construir rankings de artículos (PaperRank) y de páginas web (PageRank) y formularon una propuesta para corregir dichas debilidades. Hagen (2014) analizó los efectos de establecer un tratamiento igual (ponderación) a autores principales y co-autores; estableciendo que las métricas de los co-autores pueden llegar a ser superiores a las de los autores principales. Schreiber (2014) estudió las debilidades y dificultades de la comparación inter-temporal al aplicar el P100, que corresponde a una escala de calificación basada en los percentiles.

La siguiente tendencia, es una de las más tradicionales y corresponde a la aplicación de bibliometría a revistas o publicaciones de un área específica. Silva Andrade et al. (2016) utiliza la sociometría (análisis de redes) para analizar los "turning points" de 103 artículos en el área de la estrategia. Gregorio, Rativa \& Peralta (2015) analizan las revistas colombianas en el campo de las ciencias sociales empleando el Journal Citation Report (JCR) de ISI Web of Science (WoS). Por su parte, Valcárcel de Laiglesia et al. (2014) realizaron el estudio para 4 revistas de biomedicina; Alonso et al. (2014) analizaron las publicaciones derivadas de los congresos anuales de la Sociedad Española de Cardiología, de 2002, 2005 y 2008; Koc \& Boz (2014) elaboran el estudio para las 3 revistas más importantes en turismo entre 2003 y 2012 (Annals of Tourism Research, Tourism Management and Journal of Travel Research); Joyce, Kelly \& Sugrue (2014) trabajaron 27 revistas para identificar los 100 artículos con mayor impacto en el tema de quemaduras. Melo (2013) utilizó los artículos de la revista Gestão \& Regionalidade entre 2005 y 2012 para su trabajo. 
El último grupo de trabajos que se puede identificar es el relacionado con la evaluación de la producción en país específico. Ponomariov \& Toivanen (2014) estudiaron el papel de las capacidades nacionales de conocimientos para Brasil entre 2005-2009, identificando el ascenso de la investigación de Brasil y el incremento en la citación. De Oliveira et al. (2014) analizan la producción científica brasileña sobre dificultades de aprendizaje entre 2001 y 2011. Veiga et al. (2014) estudian la dinámica de las publicaciones nacionales, con respecto a la cirugía de rodilla entre 2000 y 2011. Si Niu (2014) trabaja a partir de la colaboración científica internacional de entre Australia y China.

\subsection{Mujer y academia}

Los trabajos sobre la relación entre mujeres y educación han ido en aumento en años recientes. Por ejemplo, Eraso (2016) mediante una investigación histórica analiza la participación de la mujer en la historia colombiana enfocándose en la medicina. Por su parte, Caputo, Vargas \& Requena (2016) y Requena, Vargas \& Caputo (2016) encuentran evidencia de que la participación de las mujeres en la academia venezolana está llegando a un estado estacionario, el cual depende de la carrera universitaria elegida, y que la brecha entre géneros tiene una tendencia a desaparecer, según la evidencia de los últimos 20 años. De Pablos \& Gil (2011) analizan para el caso español la posición de las mujeres españolas en términos de stock educativo y de resultados en el mercado laboral, comparándolo con resultados de países europeos. Así mismo, estudian el impacto en el bienestar de las mujeres de su nivel educativo de educación superior. Lemarchand (2010) a partir de cifras de la UNESCO (2009), analiza las estadísticas de participación de mujeres en el número total de investigadores del planeta. Lemarchand encuentra que a nivel mundial, para 2009, esta proporción era 29\%, pero en América Latina era de $46 \%$.

Papadópulos \& Radakovich (2006) identificaron para América Latina que hasta 2003, había una feminización incipiente en Bolivia y Perú; la feminización lograba la equidad en la matrícula en Colombia, Chile, Costa Rica, Cuba, El Salvador y México; la feminización era media en Argentina, Brasil y Venezuela; y la feminización era alta en Uruguay, República Dominicana y Panamá.

Igualmente, son múltiples los documentos que analizan la relación entre docencia y género. Por ejemplo, el trabajo de Lozano, Iglesias \& Martínez (2016) analiza la brecha de género en la Universidad de Alicante, encontrando que la presencia de académicas sigue siendo muy limitada, a pesar de las reformas universitarias sobre igualdad. Por su parte, Zapata (2010) sobre equidad de género y personal científico en universidades alemanas; Bustos \& Blázquez (2003), Buquet et al. (2006), Buquet, Cooper \& Rodríguez (2010) y Buquet, Hernández \& Jiménez (2011), para el caso de la UNAM y sus diferentes dependencias; García (1992) sobre las características socio-demográficas de las mujeres académicas y análisis de género en la Universidad de Guadalajara (García, 2004, 2009; Escolano, 2006, 2009), para el rol de las académicas en caso de las universidades españolas; Bailyn (2003) sobre las mujeres académicas en el MIT, entre otros. Asimismo, Estébañez (2007) y Gálvez (2009) identifican el fenómeno de las "tijeras", relacionado con una tendencia inversa de la presencia femenina y la evolución en la formación académica, en la formación universitaria las mujeres en conjunto son mayoritarias, mientras que en la formación posgradual su presencia va reduciéndose.

Ahora bien, aunque la relación entre género y ciencia ha sido de interés de algunos investigadores colombianos, la mayor parte de trabajos se concentran en el análisis agregado y no en áreas específicas. Los trabajos de Tovar (2002, 2004, 2005), fueron pioneros en discutir de manera crítica los indicadores de género aplicados a estadísticas nacionales de ciencia y tecnología. El trabajo de Daza (2010) presenta un análisis descriptivo sobre estadísticas e indicadores relacionados con la presencia de hombres y mujeres en el SNCTI durante el período 2000-2010, mostrando con cifras las brechas de género que aún persisten. Igualmente, Daza \& Pérez (2008) reflexionaron sobre los indicadores de género, en el campo de la ciencia y la tecnología a la luz de estudios latinoamericanos, proponiendo 4 estrategias para el caso colombiano: (i) reformular 
las preguntas y objetivos de los indicadores de género tradicionales; (ii) elaboración de indicadores de redes sociales y otros relacionados con lo que ocurre al interior de los grupos sociales; (iii) elaboración de encuestas de percepción pública de la ciencia y análisis cuantitativos de consumo y difusión de la ciencia; (iv) incluir la perspectiva de género en las políticas públicas.

Por su parte, Albert, González \& Mora (2011) estudiaron los determinantes (sexo, el nivel educativo y la situación en el mercado laboral de los padres, la composición familiar y la renta) de la demanda de educación universitaria en Colombia con datos de las Encuestas de hogares (ENH y ECH). Identifican que el porcentaje de mujeres en la universidad colombiana en el primer lustro del siglo XXI era mayor que el de hombres, situación que era contraria a la presentada en el decenio de $1980^{4}$. No obstante, también hay trabajos que analizan el "efecto tijera" en Colombia (OCyT, 2009). Pérez (2012) indicó que en 2011, el 64\% de los graduandos colombianos eran mujeres, sin embargo, de ellas solamente el $34 \%$ accede a maestrías y $20 \%$ al doctorado.

\subsection{Mujer y bibliometría}

Como se mencionó anteriormente, los trabajos que incluyen los análisis de género en los análisis bibliométricos son pocos. A continuación se hace un recuento de algunos de los más recientes.

Sos Peña (2015) analiza el impacto de 8 psicólogas norteamericanas pioneras, empleando la bibliometría para estudiar su influencia en Social Sciences Citation Index, (SSCI), entre 1900 y 1999. La autora logró identificar su importancia y alta citación en campos como: psicología infantil, historia de la psicología y lenguaje.

Ortega et al. (2015) realizaron un estudio bibliométrico sobre el papel de la mujer en 333 tesis doctorales de ciencias del deporte en España, conside-

4 Este fenómeno de feminización de la matrícula femenina también es abordado por: Albert (2000), quien señala un aumento de la participación de las mujeres en la educación superior para España; Correa (2003) para Colombia; y Bonilla et al. (2005) para Puerto Rico. rando variables como: autoría, dirección, miembros del tribunal y presidencia de tribunal, identificando un alto grado de masculinización en las cuatro variables mencionadas.

Por su parte Benoit (2015) a partir de las publicaciones del Observatoire de sciences et de technologies (OST) y los artículos de la Public Library of Science (PLoS), en total 85.131 artículos, identificó que la participación femenina es cercana al 35\% solamente y analizó el efecto "Cenicienta", según el cual, las mujeres deben hacer más esfuerzos para ser reconocidas como autores acreditados.

Tomei et al. (2014) analizan mediante el índice $\mathrm{H}$ para evaluar si existían diferencias de género de acuerdo con el rango académico y la productividad de la investigación entre los neurocirujanos hombres y mujeres. Los análisis se hicieron a partir de la información de 1.052 cirujanos de 84 instituciones diferentes. Se identificó que los hombres tenían estadísticamente mayor productividad de la investigación (en promedio un índice $\mathrm{H}$ de 13,3) que sus colegas mujeres (en promedio un índice $\mathrm{H}$ de 9,5).

En el área de la salud, también se encuentra el estudio de López et al. (2014b). Este trabajó estableció que las mujeres académicas en oftalmología siguen estando sub-representadas entre los profesores titulares. Aunque las mujeres superan a los hombres en la productividad académica durante las últimas etapas de sus carreras, el bajo impacto académico de su producción durante las primeras etapas puede impedir su avance académico y explicar, en parte, la disparidad de género en altos puestos académicos.

El trabajo de Brooks, Fenton \& Walker (2014) empleó los datos de las escuelas de negocios del Reino Unido, encontrando que hay diferencias importantes entre la calidad de las revistas en las cuales publican hombres y mujeres, en las áreas de gestión $y$ contabilidad. Igualmente, identificaron que las mujeres que son capaces de utilizar las redes de coautor con personas fuera de su institución publican en las revistas de mayor calificación. 
Por su parte, Abramo, D’Angelo \& Murgia (2013) analizan el rol de las mujeres académicas italianas en la colaboración científica. Los autores analizan la propensión a la colaboración de los académicos según los campos, disciplinas y formas de colaboración: intramural, extramural nacional e internacional. Sus resultados indicaron que las mujeres investigadoras registran una mayor capacidad de colaborar en todas las formas analizadas, con excepción de la colaboración internacional, donde todavía hay una brecha en comparación con sus colegas masculinos.

A su vez, Weiss, Kovshilovskaya \& Breyer (2012) identificaron que la tasa de crecimiento de las publicaciones y la visibilidad de la producción académica de las mujeres en urología era superior a la tasa de crecimiento de la presencia de mujeres en el área. Concluyen que las mujeres en urología son miembros activos y productivos de la comunidad académica en urología.

Aleixandre et al. (2007) analizan la paridad de la autoría de los artículos publicados en Enfermedades Infecciosas y Microbiología Clínica (EIMC). A partir de los registros del Science Citation Index-Expanded 1 entre 2001-2005. De los 2.163 autores, 943 fueron mujeres $(43,6 \%)$, la presencia y la productividad de las mujeres en las instituciones más reconocidas fue más baja que las de los hombres.

Finalmente, Kretschmer \& Aguillo (2005) desarrollaron tres indicadores aplicadas a redes académicas en internet: cooperación de género, tasa de visibilidad web y centralidad de género en las redes. Establecieron que en la red analizada Collnet, no hay diferencias significativas en la colaboración, pero la visibilidad es mayor para aquellos trabajos de coautores masculinos únicamente.

Después de haber establecido las tendencias más representativas en la producción sobre bibliometría y enfoque de género, se procederá a realizar el análisis para identificar los patrones de la producción colombiana en economía y administración.

\section{Indicadores básicos}

\subsection{Datos y metodología}

El primer paso para iniciar el ejercicio de bibliometría consistió en seleccionar el índice bibliográfico a utilizar. Se eligió Scopus y no ISI, dado que la cobertura de países latinoamericanos es mayor en el primer índice. En segundo lugar se estableció como criterio de búsqueda en Scopus el siguiente algoritmo: AFFILCOUNTRY (Colombia) AND (LIMIT-TO(SUBJAREA, "ECON") OR LIMIT-TO (SUBJAREA, "BUSI"). Esto quiere decir que la búsqueda arrojó documentos de autores cuya filiación correspondía a una institución que se encontrara en Colombia y que hubieran publicado en el área de economía (economics, econometrics and finance - EEF) o administración (business, management and accounting - BMA). A continuación, se llevó a cabo una depuración para verificar que todos los documentos pertenecieran a autores colombianos. Posteriormente, se adicionó el número de autores y autoras, y se calculó el porcentaje de mujeres dentro del total de autores. Así mismo, se estableció si la mujer era autora principal o no. Finalmente, se inició el análisis de los indicadores que se presenta a continuación.

\subsection{Volumen de la producción}

El número de documentos indexados en Scopus del área de $\mathrm{BMA}^{5}$ fue 410, de EEF 758 y de ambas disciplinas 145, en los cuales participó al menos un autor de origen colombiano. Como se aprecia en la Ilustración 1, las publicaciones en BMA solamente adquieren continuidad desde 1995, alcanzando su valor máximo en 2013 (97 publicaciones ${ }^{6}$ ). En el caso de las publicaciones de EEF, el mayor valor alcanzado se presenta también en 2013, con 144 productos $^{7}$.

5 Esta clasificación corresponde a la dada por Scopus a la publicación en la cual se difundió el trabajo.

6 Incluyendo las publicaciones que pertenecen a ambas categorías.

7 Incluyendo las publicaciones que pertenecen a ambas categorías. 
Ilustración 1. Consolidado del volumen de producción académica en las áreas de BMA y EEF (enero de 1974-junio de 2014)

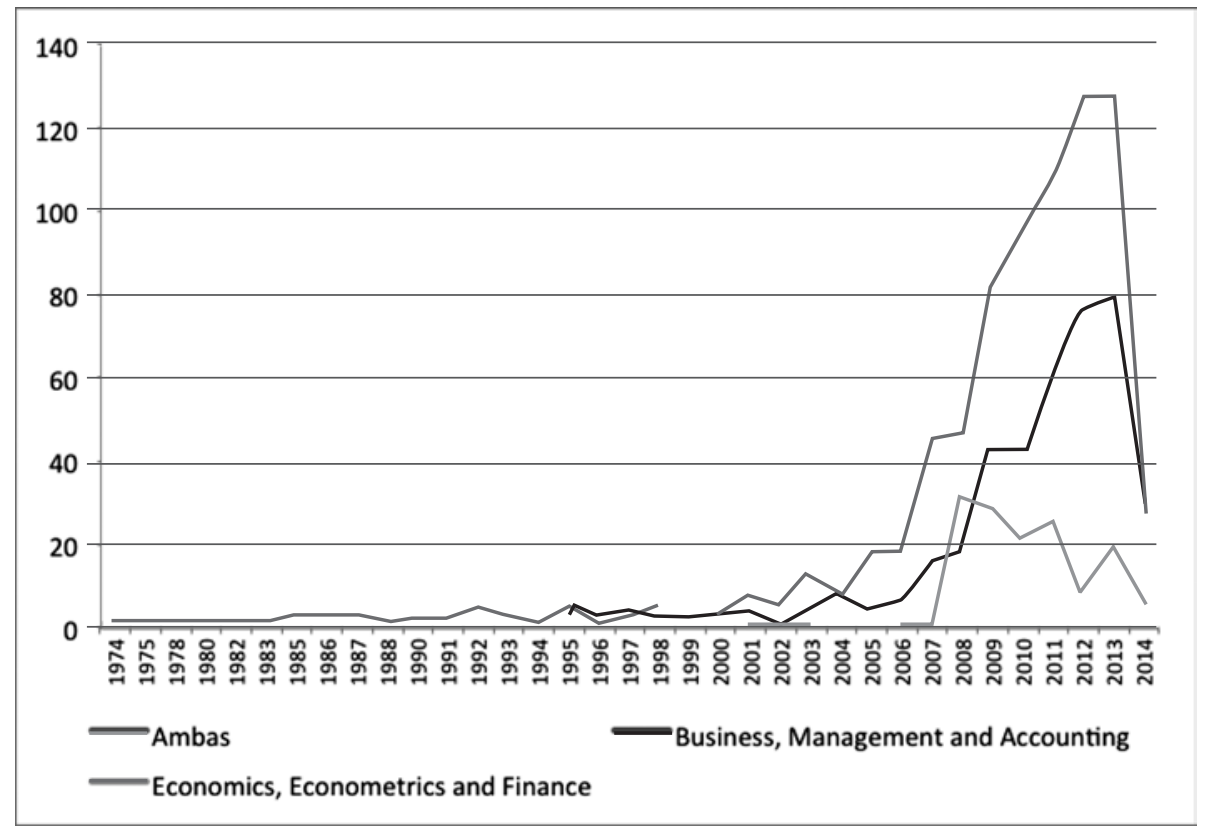

Fuente: Elaboración propia a partir de los datos de Scopus.

$\mathrm{Al}$ analizar el volumen de producción, dependiendo de la presencia de las mujeres como autoras, se encuentra que en el agregado, el 60,2\% de los trabajos fueron escritos por hombres, sin participación de ninguna mujer. Solamente en el 21,78\% de los artículos las mujeres fueron las autoras principales.
En economía el porcentaje de artículos escritos sólo por hombres es mayor (64,6\%). El área con mayor tasa de autoras principales es BMA con $26,3 \%$ (Tabla 1 e Ilustración 2).

Tabla 1. Volumen de producción académica en las áreas de BMA y EEF, por sexo (enero de 1974-junio de 2014)

\begin{tabular}{|l|c|c|c|c|c|c|c|c|}
\hline & Ambas & $\%$ & BMA & $\%$ & EEF & $\%$ & Total & $\%$ \\
\hline No hay autoras mujeres & 82 & $56,60 \%$ & 216 & $52,70 \%$ & 490 & $64,60 \%$ & 788 & $60,02 \%$ \\
\hline Hay autoras mujeres, pero no son principales & 36 & $24,80 \%$ & 86 & $21,00 \%$ & 117 & $15,40 \%$ & 239 & $18,20 \%$ \\
\hline El autor principal es mujer & 27 & $18,60 \%$ & 108 & $26,30 \%$ & 151 & $19,90 \%$ & 286 & $21,78 \%$ \\
\hline Total general & $\mathbf{1 4 5}$ & $100,00 \%$ & $\mathbf{4 1 0}$ & $100,00 \%$ & $\mathbf{7 5 8}$ & $100 \%$ & $\mathbf{1 . 3 1 3}$ & $100 \%$ \\
\hline
\end{tabular}

Fuente: Elaboración propia a partir de los datos de Scopus. 
Ilustración 2. Volumen de producción académica en las áreas de BMA y EEF, por sexo (enero de 1974-junio de 2014)

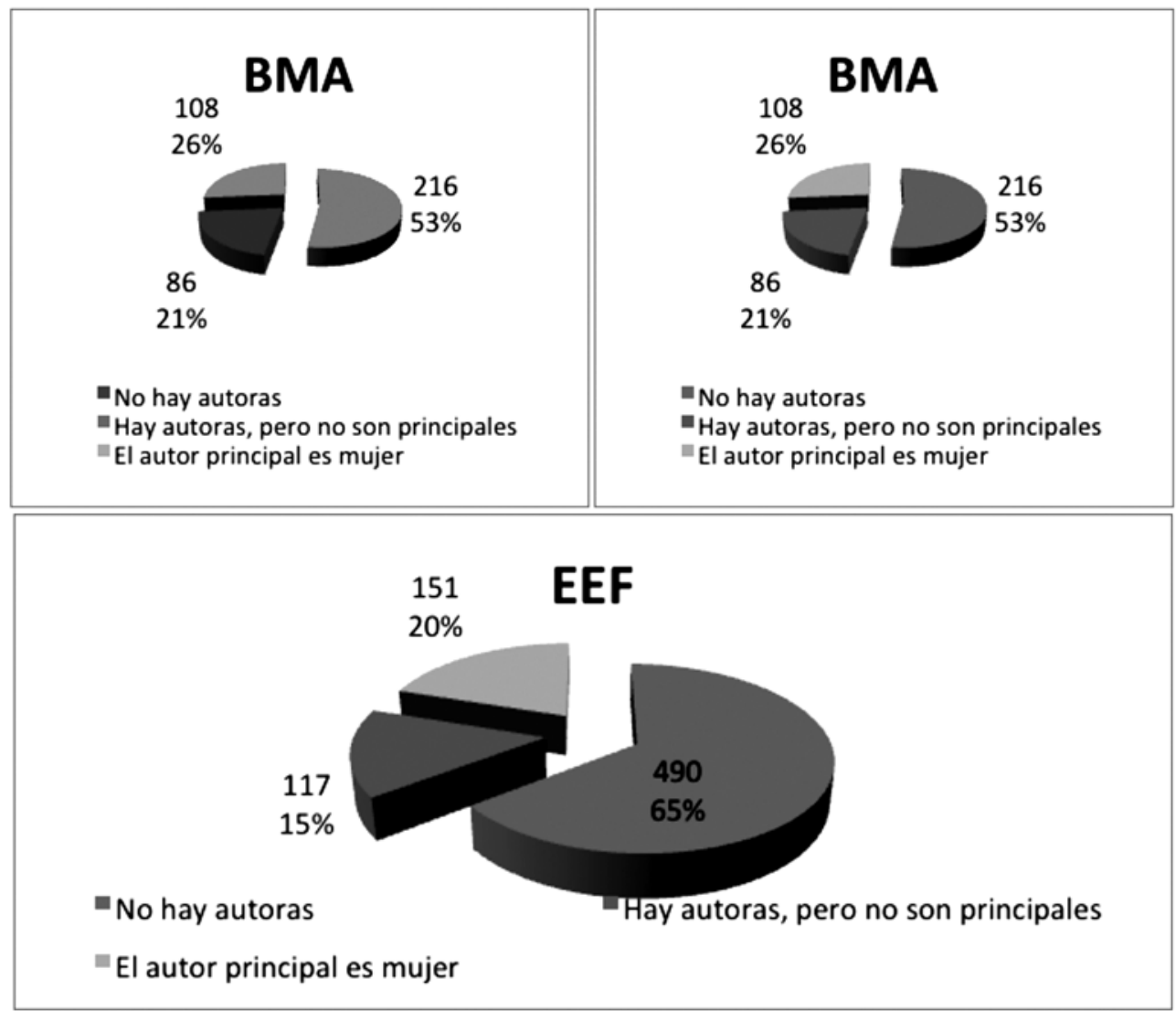

Fuente: elaboración propia a partir de los datos de Scopus.

En la Ilustración 3 se aprecia que las publicaciones de hombres, sin la participación de mujeres siempre han sido superiores a las publicaciones de las mujeres; la diferencia se acentúa a partir de 2005. No obstante lo anterior, al considerar la tasa de crecimiento anual (Ilustración 4), se aprecia que la incorporación de mujeres en la producción académica se ha incrementado más que la participación de los hombres.

Una mirada por tipología de los documentos, muestra que la mayor parte de trabajos en las áreas de BMA y EEF corresponde a artículos publicados: $90,34 \%$ y $77,07 \%$ respectivamente, en el caso de ambas disciplinas esta participación es de 91,03\%.
La segunda mayor participación en BMA es documentos de conferencia $(7,80 \%)$, mientras que para EEF son los reviews (3,69\%). En la categoría otros se incluyen notas, erratas, editoriales, cartas al editor, y short review (Tabla 3).

En la Tabla 2 también puede apreciarse que el área en la cual hay mayor paridad en la autoría de artículos, corresponde al BMA en la cual 50,78\% de los trabajos son escritos por hombres sin la participación de mujeres. Por su parte, el área de EEF presenta un comportamiento masculinizado, ya que 63,39\% de los artículos durante el período fueron escritos por hombres únicamente. 
Ilustración 3. Evolución del volumen de producción académica en las áreas de BMA y EEF, por sexo (enero de 1974-junio de 2014)

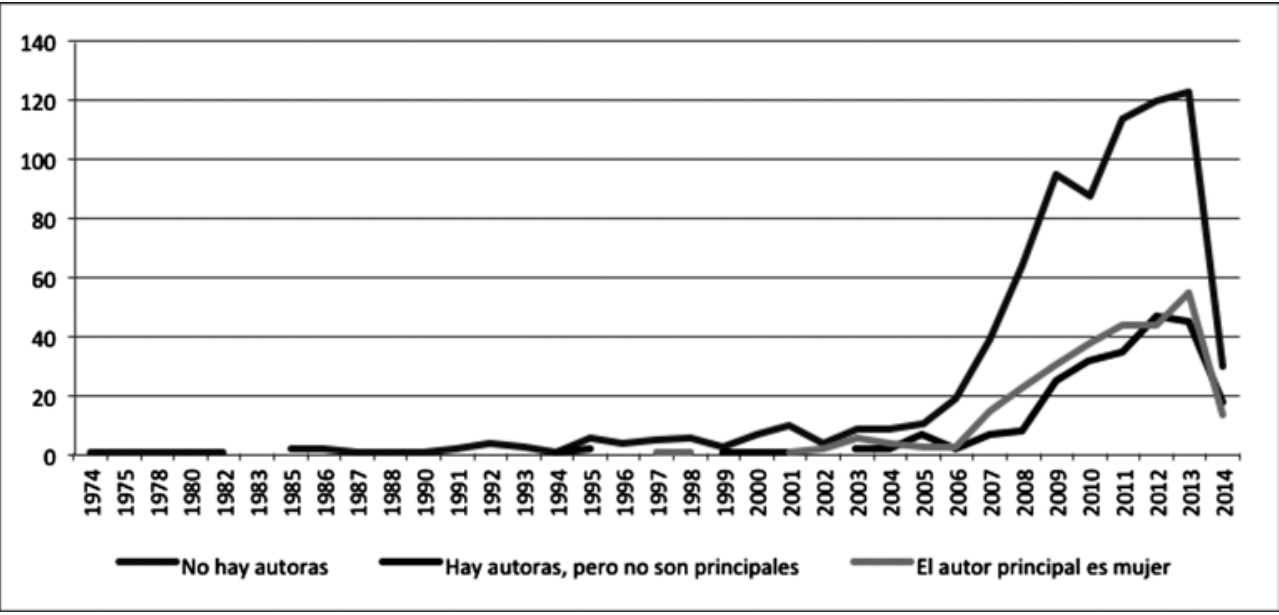

Fuente: Elaboración propia a partir de los datos de Scopus.

Ilustración 4. Tasa de crecimiento del volumen de producción académica en las áreas de BMA y EEF, por sexo (enero de 1974-junio de 2014)

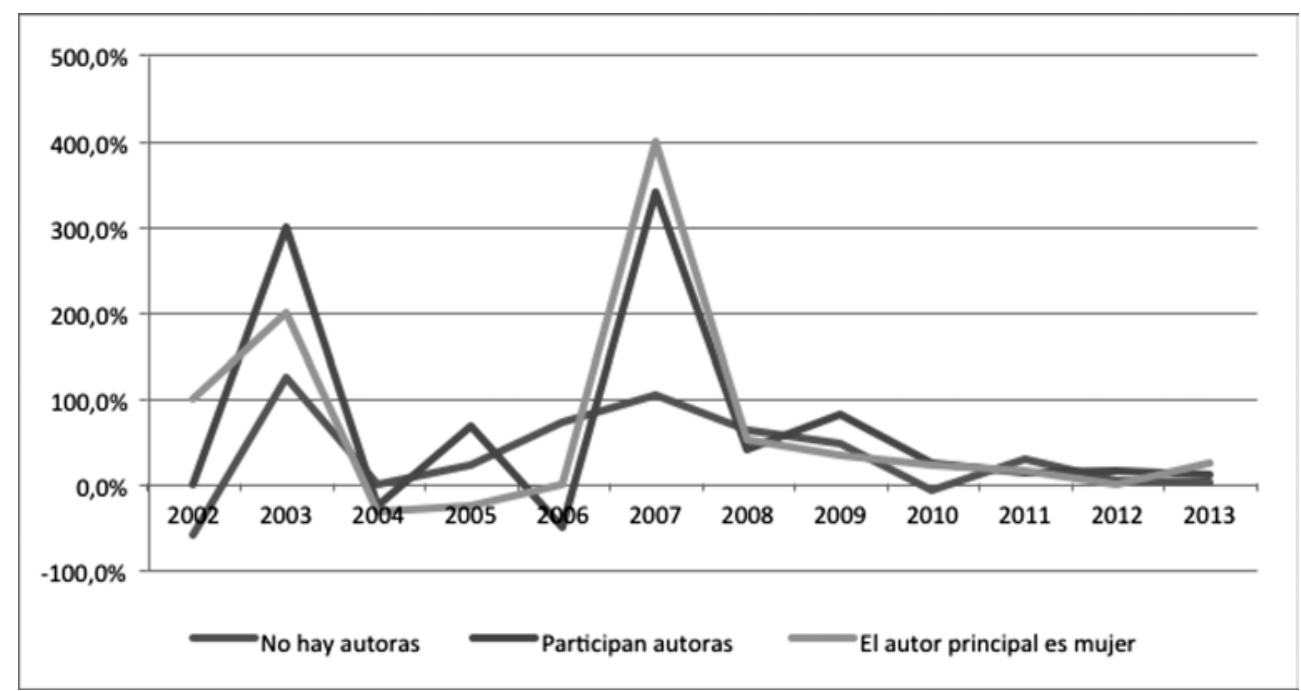

Fuente: Elaboración propia a partir de los datos de Scopus. 
Tabla 2. Tipos de documentos Fuente: elaboración propia a partir de los datos de Scopus

\begin{tabular}{|c|c|c|c|c|}
\hline & No hay autoras mujeres & Hay autoras mujeres, pero no son principales & El autor principal es mujer & Total \\
\hline Ambas & 82 & 36 & 27 & 145 \\
\hline Artículos & 79 & 29 & 24 & 132 \\
\hline Conferencia & 2 & 2 & & 4 \\
\hline Review & 1 & 5 & 3 & 9 \\
\hline BMA & 216 & 86 & 108 & 410 \\
\hline Artículos & 162 & 70 & 87 & 319 \\
\hline Capítulo de libro & 7 & 1 & 3 & 11 \\
\hline Conferencia & 15 & 7 & 10 & 32 \\
\hline Review & 12 & 8 & 3 & 23 \\
\hline Otros & 20 & 0 & 5 & 25 \\
\hline EEF & 490 & 117 & 151 & 758 \\
\hline Artículos & 444 & 109 & 142 & 695 \\
\hline Capítulo de libro & 5 & & 2 & 7 \\
\hline Conferencia & 3 & 2 & 2 & 7 \\
\hline Otros & 17 & 2 & 1 & 20 \\
\hline Review & 21 & 4 & 4 & 29 \\
\hline Total & 788 & 239 & 286 & 1.313 \\
\hline
\end{tabular}

Fuente: Elaboración propia a partir de los datos de Scopus.

\subsection{Fuentes}

Considerando de manera agregada el área de ciencias económicas, la revista con mayor número de artículos de autores con al menos uno de origen colombiano es Cuadernos de Economía con 113 artículos, seguida de Cuadernos de Administración con 98 trabajos. Cabe resaltar que las primeras 8 posiciones son ocupadas por revistas colombianas: Cuadernos de Economía, Cuadernos de Administración, Innovar, Revista de Economía Institucional, Ensayos Sobre Política Económica, Revista de Economía del Rosario, Desarrollo y Sociedad y Cuadernos de Desarrollo Rural (Tabla 3).

De acuerdo con la Tabla 4, en términos absolutos, la revista en la cual más artículos con participación de mujeres han sido publicados durante el período, fue Cuadernos de Administración (98 trabajos, $42,9 \%$ ), seguido de la revista Innovar (87 trabajos, $42,5 \%)$. Las revistas que se encuentran en la Tabla 5 concentran 44,5\% del total de artículos en los cuales hay autores femeninos (234 de 525), y el $49 \%$ de los artículos cuya autora principal es una mujer (140 de 286).

Así mismo, debe mencionarse que dentro de las revistas con mayor participación femenina también se encuentran las revistas que en proporción mayor número de artículos de autores hombres han difundido. Tales son los casos de Economía Institucional (80\%), Cuadernos de Economía (78,6\%), Journal of Development Economics (75\%), Revista de economía del Rosario $(74,3 \%)$ y Desarrollo y Sociedad (73,5\%). 
Tabla 3. Principales revistas que publicaron artículos en EEF o BMA (enero de 1974 - junio de 2014) ${ }^{8}$

\begin{tabular}{|c|c|c|c|c|}
\hline Revista & País & Ambas & $\begin{array}{l}\text { Business, Management } \\
\text { and Accounting }\end{array}$ & $\begin{array}{c}\text { Economics, Econometrics } \\
\text { and Finance }\end{array}$ \\
\hline Cuadernos de Economía & Colombia & & & 113 \\
\hline Cuadernos de Administración & Colombia & 98 & & \\
\hline Innovar & Colombia & & 87 & \\
\hline Revista de Economía Institucional & Colombia & & & 85 \\
\hline Ensayos Sobre Política Económica & Colombia & & & 50 \\
\hline Revista de Economía del Rosario & Colombia & & & 35 \\
\hline Desarrollo y Sociedad & Colombia & & & 34 \\
\hline Cuadernos de Desarrollo Rural & Colombia & & & 31 \\
\hline Journal of Development Economics & Holanda & & & 24 \\
\hline Ecological Economics & Holanda & & & 17 \\
\hline Academia & Venezuela & & 15 & \\
\hline Journal of Business Research & Holanda & & 12 & \\
\hline Journal of Technology Management and Innovation & Chile & & 11 & \\
\hline Journal of the Operational Research Society & Reino Unido & & 10 & \\
\hline Economic Modelling & Holanda & & & 9 \\
\hline Journal of Cleaner Production & Holanda & & 9 & \\
\hline Banking Law Journal & Estados Unidos & 8 & & \\
\hline Energy Economics & Holanda & & & 8 \\
\hline Food Policy & Estados Unidos & & & 8 \\
\hline
\end{tabular}

Fuente: Elaboración propia a partir de los datos de Scopus.

La mayor parte de las revistas que concentran las publicaciones de mujeres son colombianas. De las 15 revistas que se muestran en la Tabla 4,8 son colombianas.

Hay 148 fuentes que han publicado solamente trabajos de autores hombres en ciencias económicas.

Cabe mencionar que:

- La mayor parte de las revistas que concentran las publicaciones de mujeres son colombianas.
De las 15 revistas que se muestran en la Tabla 4,8 son colombianas.

- Hay 148 fuentes que han publicado solamente trabajos de autores hombres en ciencias económicas.

- Hay 45 fuentes que sólo han publicado trabajos en los cuales hay autoras. Algunas de dichas publicaciones están relacionadas con salud, turismo y ciencias agronómicas. 
Tabla 4. Revistas con mayor número de artículos en los que hayan participado al menos una mujer (enero de 1974 junio de 2014)

\begin{tabular}{|c|c|c|c|c|c|c|c|c|c|}
\hline Revista & $\begin{array}{l}\text { No hay } \\
\text { autoras }\end{array}$ & $\begin{array}{l}\text { Hay autoras } \\
\text { pero no son } \\
\text { principales }\end{array}$ & $\begin{array}{l}\text { El autor } \\
\text { principal } \\
\text { es mujer }\end{array}$ & $\begin{array}{l}\text { Participa } \\
\text { al menos } \\
\text { una mujer }\end{array}$ & Total & $\begin{array}{l}\text { No hay } \\
\text { autoras } \\
(\%)\end{array}$ & $\begin{array}{l}\text { Hay autoras } \\
\text { pero no son } \\
\text { principales } \\
(\%)\end{array}$ & $\begin{array}{c}\text { El autor } \\
\text { principal } \\
\text { es mujer } \\
(\%)\end{array}$ & $\begin{array}{c}\text { Participa al } \\
\text { menos una } \\
\text { mujer (\%) }\end{array}$ \\
\hline $\begin{array}{l}\text { Cuadernos de } \\
\text { Administración }\end{array}$ & 56 & 19 & 23 & 42 & 98 & $57,1 \%$ & $42,9 \%$ & $23,5 \%$ & $42,9 \%$ \\
\hline Innovar & 50 & 13 & 24 & 37 & 87 & $57,5 \%$ & $42,5 \%$ & $27,6 \%$ & $42,5 \%$ \\
\hline $\begin{array}{l}\text { Cuadernos de } \\
\text { Economia }\end{array}$ & 81 & 14 & 18 & 32 & 103 & $78,6 \%$ & $31,1 \%$ & $17,5 \%$ & $31,1 \%$ \\
\hline $\begin{array}{l}\text { Ensayos Sobre } \\
\text { Política Económica }\end{array}$ & 30 & 8 & 12 & 20 & 50 & $60,0 \%$ & $40,0 \%$ & $24,0 \%$ & $40,0 \%$ \\
\hline $\begin{array}{l}\text { Revista de } \\
\text { Economia Institucional }\end{array}$ & 68 & 7 & 10 & 17 & 85 & $80,0 \%$ & $20,0 \%$ & $11,8 \%$ & $20,0 \%$ \\
\hline $\begin{array}{l}\text { Cuadernos de } \\
\text { Desarrollo Rural }\end{array}$ & 15 & 2 & 14 & 16 & 31 & $48,4 \%$ & $51,6 \%$ & $45,2 \%$ & $51,6 \%$ \\
\hline World Development & 14 & 4 & 7 & 11 & 25 & $56,0 \%$ & $44,0 \%$ & $28,0 \%$ & $44,0 \%$ \\
\hline $\begin{array}{l}\text { Journal of Technology } \\
\text { Management and } \\
\text { Innovation }\end{array}$ & 1 & 4 & 6 & 10 & 11 & $9,1 \%$ & $90,9 \%$ & $54,5 \%$ & $90,9 \%$ \\
\hline $\begin{array}{l}\text { Revista de Economia } \\
\text { del Rosario }\end{array}$ & 26 & 3 & 6 & 9 & 35 & $74,3 \%$ & $25,7 \%$ & $17,1 \%$ & $25,7 \%$ \\
\hline Desarrollo y Sociedad & 25 & 5 & 4 & 9 & 34 & $73,5 \%$ & $26,5 \%$ & $11,8 \%$ & $26,5 \%$ \\
\hline $\begin{array}{l}\text { Journal of Business } \\
\text { Research }\end{array}$ & 4 & 5 & 3 & 8 & 12 & $33,3 \%$ & $66,7 \%$ & $25,0 \%$ & $66,7 \%$ \\
\hline Ecological Economics & 10 & 2 & 5 & 7 & 17 & $58,8 \%$ & $41,2 \%$ & $29,4 \%$ & $41,2 \%$ \\
\hline $\begin{array}{l}\text { Journal of Develop- } \\
\text { ment Economics }\end{array}$ & 18 & 4 & 2 & 6 & 24 & $75,0 \%$ & $25,0 \%$ & $8,3 \%$ & $25,0 \%$ \\
\hline Academia & 10 & 2 & 3 & 5 & 15 & $66,7 \%$ & $33,3 \%$ & $20,0 \%$ & $33,3 \%$ \\
\hline $\begin{array}{l}\text { Peace Economics, } \\
\text { Peace Science and } \\
\text { Public Policy }\end{array}$ & 3 & 2 & 3 & 5 & 8 & $37,5 \%$ & $62,5 \%$ & $37,5 \%$ & $62,5 \%$ \\
\hline
\end{tabular}

Fuente: Elaboración propia a partir de los datos de Scopus.

Ahora bien, el número total de fuentes en el período analizado asciende a 357 , pero aquellas que han publicado entre uno y dos documentos (268) concentran el $25,97 \%$ del total de la producción publicada, lo que equivale a 341 trabajos. Visto desde otro enfoque las primeras 10 revistas (8 de las cuales son colombianas) centralizan el $44,33 \%$ de la producción académica en ambas disciplinas (582 artículos). Al considerar de manera independiente los autores hombres y mujeres (Tabla 5 e Ilustración 5), la tendencia a que las publicaciones con uno o dos artículos concentren la mayor parte de trabajos, 
se mantiene. Para el caso de la mujer como autora principal corresponde al 47,55\%, para al menos una mujer como autora es el $41,9 \%$ y para sólo autores hombres es del 30,96\%.

\subsection{Idioma de publicación}

Como se aprecia en la Tabla 6, el idioma de publicación más relevante en el agregado es el inglés. No obstante, al considerar el número de documentos que fueron publicados en revistas que abarcan las dos áreas, el idioma más frecuente es español. Otro elemento a considerar es que en EEF se publicaron documentos en francés, mientras que en BMA no fue así. No obstante, en BMA hay trabajos publicados en portugués, húngaro y croata.

El mismo comportamiento, se presenta al analizar la segmentación por sexo. El 71\% de los artículos escritos sólo por hombres fueron escritos en inglés.
El 79\% de los trabajos donde participan mujeres, pero no son autoras principales, fueron escritos en este mismo idioma. Para el caso de las autoras principales esta cifra asciende al $76 \%$. El segundo idioma de mayor publicación es español (Tabla 7).

\subsection{Autores}

Dentro de los autores con mayor producción sólo se encuentran dos mujeres: Ana María Iregui del Banco de la República y Jennifer Lafont de la Universidad de Córdoba (Tabla 8). En los 1.313 trabajos analizados, participaron 3.156 autores, de los cuales sólo 756 eran mujeres, es decir el 24\%. En las publicaciones multidisciplinarias la participación de las mujeres fue de $25,1 \%$, en BMA $26,9 \%$ y en EEF 21,6\%. En otras palabras, en el agregado se presenta un fenómeno de masculinización de la producción académica de autores colombianos, en ciencias económicas.

Ilustración 5. Número de documentos publicados sobre EEF y BMA por fuente (enero de 1974-junio de 2014)

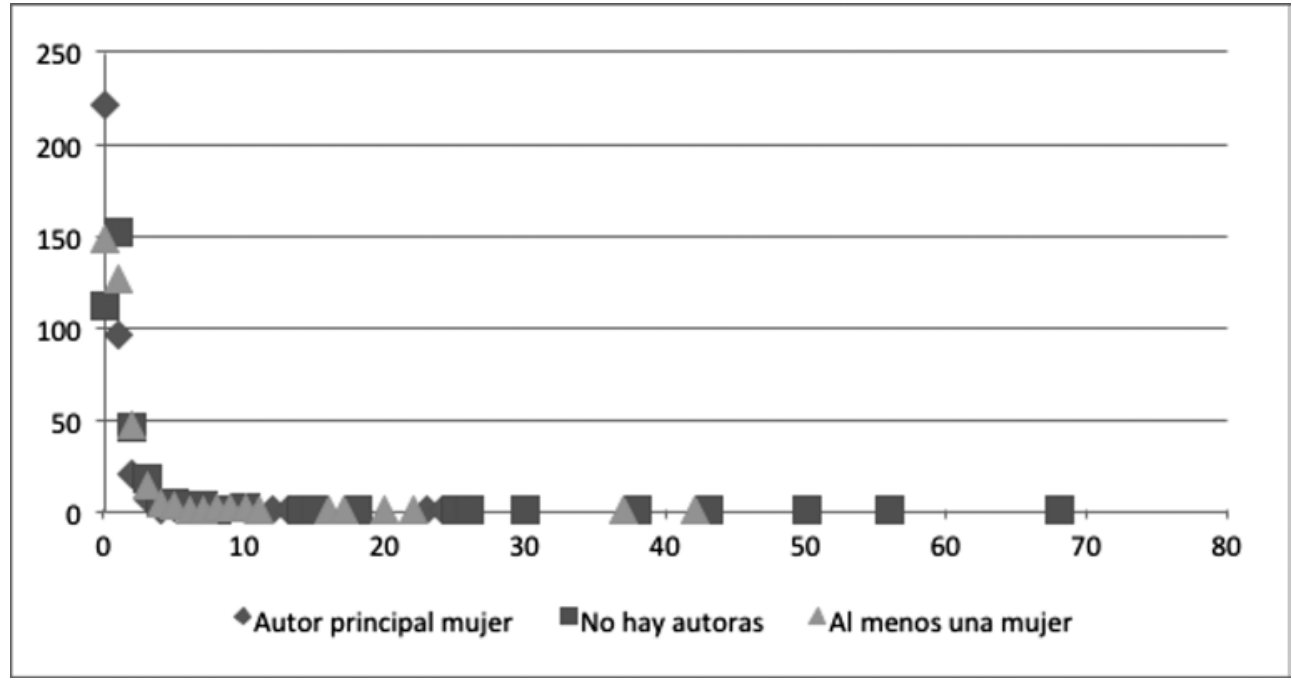

Fuente: Elaboración propia a partir de los datos de Scopus. 
Tabla 5. Número de documentos publicados sobre EEF y BMA por fuente (enero de 1974-junio de 2014)

\begin{tabular}{|c|c|c|c|}
\hline \multirow[b]{2}{*}{ Número de documentos } & \multicolumn{3}{|c|}{ Fuentes } \\
\hline & $\begin{array}{c}\text { Autor principal } \\
\text { mujer }\end{array}$ & No hay autoras & Al menos una mujer \\
\hline 0 & 222 & 112 & 148 \\
\hline 1 & 96 & 152 & 126 \\
\hline 2 & 20 & 46 & 47 \\
\hline 3 & 7 & 18 & 14 \\
\hline 4 & 1 & 5 & 4 \\
\hline 5 & 2 & 4 & 3 \\
\hline 6 & 2 & 3 & 1 \\
\hline 7 & 1 & 3 & 1 \\
\hline 8 & & 1 & 2 \\
\hline 9 & & & 2 \\
\hline 10 & 1 & 2 & 2 \\
\hline 11 & & & 1 \\
\hline 12 & 1 & & \\
\hline 13 & 1 & & \\
\hline 14 & 1 & 1 & \\
\hline 15 & & 1 & \\
\hline 16 & & & 1 \\
\hline 17 & & & 1 \\
\hline 18 & & 1 & \\
\hline 20 & & & 1 \\
\hline 22 & & & 1 \\
\hline 23 & 1 & & \\
\hline 24 & 1 & & \\
\hline 25 & & 1 & \\
\hline 26 & & 1 & \\
\hline 30 & & 1 & \\
\hline 37 & & & 1 \\
\hline 38 & & 1 & \\
\hline 42 & & & 1 \\
\hline 43 & & 1 & \\
\hline 50 & & 1 & \\
\hline 56 & & 1 & \\
\hline 68 & & 1 & \\
\hline
\end{tabular}

Fuente: elaboración propia. 
Tabla 6. Idioma de elaboración de los trabajos publicados sobre EEF y BMA por fuente (enero de 1974-junio de 2014)

\begin{tabular}{|l|c|c|c|c|}
\hline & Ambas & $\begin{array}{c}\text { Business, Management and } \\
\text { Accounting }\end{array}$ & $\begin{array}{c}\text { Economics, Econometrics } \\
\text { and Finance }\end{array}$ & Total \\
\hline Inglés & 44 & 289 & 403 & 736 \\
\hline Español & 100 & 112 & 350 & 562 \\
\hline Portugués & 1 & 7 & 0 & 8 \\
\hline Francés & & 1 & 4 & 4 \\
\hline Húngaro & & 1 & & 1 \\
\hline Croata & & 1 & & 1 \\
\hline
\end{tabular}

Fuente: Elaboración propia a partir de los datos de Scopus.

Tabla 7. Idioma de elaboración de los trabajos publicados sobre EEF y BMA por fuente (enero de 1974-junio de 2014)

\begin{tabular}{|l|c|c|c|c|}
\hline \multicolumn{1}{|c|}{ Idioma } & No hay autoras & $\begin{array}{c}\text { Hay autoras, pero no son el } \\
\text { autor principal }\end{array}$ & Autora principal & Total \\
\hline Inglés & 561 & 190 & 217 & 968 \\
\hline Español & 224 & 44 & 65 & 333 \\
\hline Croata & & & 1 & 1 \\
\hline Francés & 3 & 5 & 1 & 4 \\
\hline Húngaro & & $\mathbf{2 3 9}$ & 1 & 1 \\
\hline Portugués & $\mathbf{7 8 8}$ & & $\mathbf{2 8 6}$ & $\mathbf{1 . 3 1 3}$ \\
\hline Total & & & & 1 \\
\hline
\end{tabular}

Fuente: Elaboración propia a partir de los datos de Scopus.

\subsection{Países en los cuales se publican los trabajos de origen colombiano}

Para la elaboración de este apartado, se utilizó la información de la base de datos del total de las revistas incluidas en Scopus a junio de 2014. A partir de ella, se identificó el país de origen de la publicación en la cual se difundieron los documentos de origen colombianos. Los resultados se pueden visualizar en Ilustración 6 y la Tabla 9.
En el agregado 533 de los 1.313 artículos (40,59\%) fueron publicados en fuentes colombianas, 236 en publicaciones holandesas (17,97\%), 205 en fuentes del Reino Unido (15,61\%), 196 en revistas americanas (14,93\%). Cabe mencionar que si se analizan de forma independiente los trabajos de BMA y EEF, los resultados cambian un poco. La mayor parte de trabajos de BMA se publicó en revistas de origen estadounidense (98), seguido de Colombia (87) y Reino Unido (84). En el caso de los documentos de EEF, la mayoría se publicó en revistas colombianas (348), seguido de Holanda (157) y Reino Unido (114). 
Tabla 8. Principales autores de la producción en EEF y BMA (enero de 1974 - junio de 2014)

\begin{tabular}{|c|c|c|c|c|c|c|c|}
\hline Autor & Artículos & Afiliación & $\begin{array}{l}\text { Documentos } \\
\text { totales } \\
\text { en la base }\end{array}$ & $\begin{array}{c}\text { Índice } \\
\text { H }\end{array}$ & $\begin{array}{c}\text { Citaciones } \\
\text { totales }\end{array}$ & $\begin{array}{l}\text { Documentos de } \\
\text { la base que lo } \\
\text { citan }\end{array}$ & Coautores \\
\hline Otero, J. & 27 & Universidad del Rosario & 38 & 7 & 140 & 129 & 11 \\
\hline Cárdenas, J.C. & 19 & Universidad de los Andes & 33 & 12 & 682 & 498 & 61 \\
\hline Alvis, A. & 16 & Universidad de Córdoba & 21 & 3 & 41 & 34 & 17 \\
\hline Montoya Torres, J.R. & 13 & Universidad de la Sabana & 45 & 6 & 121 & 105 & 57 \\
\hline Ríos, L.A. & 13 & Universidad de Antioquia & 64 & 9 & 289 & 235 & 65 \\
\hline Páez, M.S. & 12 & Universidad de Córdoba & 29 & 7 & 119 & 92 & 28 \\
\hline Gómez González, J.E. & 12 & Banco de la República & 18 & 3 & 18 & 15 & 21 \\
\hline Dyner, l. & 11 & Universidad Nacional de Colombia & 30 & 9 & 238 & 225 & 50 \\
\hline Bogliacino, F. & 10 & Universidad Konrad Lorenz & 16 & 4 & 44 & 34 & 13 \\
\hline Gonzalez, A. & 10 & Banco de la República & 16 & 4 & 27 & 26 & 35 \\
\hline Duque Oliva, E.J. & 9 & Universidad Nacional de Colombia & 10 & 1 & 3 & 3 & 4 \\
\hline Vélez Pareja, I. & 9 & $\begin{array}{l}\text { Principio del formulario } \\
\text { Universidad Tecnológica de } \\
\text { BolívarFinal del formulario }\end{array}$ & 10 & 1 & 12 & 9 & 11 \\
\hline Marín, J.M. & 9 & Universidad de Antioquia & 43 & 8 & 249 & 219 & 49 \\
\hline Iregui, A.M. & 9 & Banco de la República & 10 & 2 & 13 & 13 & 5 \\
\hline Lafont, J.J. & 9 & $\begin{array}{l}\text { Principio del formulario } \\
\text { Universidad de CórdobaFinal } \\
\text { del formulario }\end{array}$ & 14 & 3 & 28 & 25 & 20 \\
\hline Alvarez, C. & 9 & $\begin{array}{l}\text { Principio del formulario } \\
\text { Universidad de MedellínFinal } \\
\text { del formulario }\end{array}$ & 14 & 2 & 15 & 13 & 9 \\
\hline Arango, L.E. & 8 & $\begin{array}{l}\text { Principio del formulario } \\
\text { Banco de la RepúblicaFinal } \\
\text { del formulario }\end{array}$ & 14 & 3 & 23 & 22 & 14 \\
\hline Ensuncho, A.E. & 8 & $\begin{array}{l}\text { Principio del formulario } \\
\text { Universidad de CórdobaFinal } \\
\text { del formulario }\end{array}$ & 11 & 3 & 29 & 17 & 12 \\
\hline Zuleta, H. & 8 & Universidad de los Andes & 14 & 4 & 37 & 26 & 6 \\
\hline Vargas, J.F. & 8 & Universidad del Rosario & 9 & 2 & 30 & 30 & 10 \\
\hline Castrillon, O.D. & 8 & Universidad Nacional de Colombia & 14 & 3 & 19 & 13 & 16 \\
\hline
\end{tabular}

Fuente: elaboración propia a partir de los datos de Scopus. 
Ahora bien, los países con mayor publicación de artículos con participación de mujeres son Colombia (182), Holanda (94), Reino Unido (91) y Estados Unidos (83). Este mismo orden se mantiene para los artículos cuyo autor principal es una mujer y para aquellos en los cuales ninguna mujer participa (Tabla 10). En publicaciones de Dinamarca y Ucrania, a pesar de que hay publicaciones en el área de autores hombres, no hay ninguna en la que al menos una autora haya participado.

Tabla 9. Países en los cuales se difunden los artículos de autores colombianos (enero de 1974 - junio de 2014)

\begin{tabular}{|c|c|c|c|c|}
\hline País & Ambas & $\begin{array}{l}\text { Business, Management } \\
\text { and Accounting }\end{array}$ & $\begin{array}{c}\text { Economics, Econometrics } \\
\text { and Finance }\end{array}$ & Total \\
\hline Alemania & 1 & 19 & 8 & 28 \\
\hline Australia & & 3 & & 3 \\
\hline Brasil & & 3 & 3 & 6 \\
\hline Canadá & & 6 & 1 & 7 \\
\hline Chile & & 15 & 13 & 28 \\
\hline Colombia & 98 & 87 & 348 & 533 \\
\hline Corea del Sur & & & 2 & 2 \\
\hline Croacia & & 2 & & 2 \\
\hline Dinamarca & & 1 & & 1 \\
\hline España & 3 & 7 & 13 & 23 \\
\hline Estados Unidos & 13 & 98 & 85 & 196 \\
\hline Filipinas & 2 & & 1 & 3 \\
\hline Francia & 1 & & 1 & 2 \\
\hline Grecia & & 1 & & 1 \\
\hline Holanda & 17 & 62 & 157 & 236 \\
\hline México & & & 8 & 8 \\
\hline Nueva Zelanda & & & 1 & 1 \\
\hline Reino Unido & 7 & 84 & 114 & 205 \\
\hline Serbia & & 1 & & 1 \\
\hline Singapore & 3 & & 1 & 4 \\
\hline Sudádrica & & & 1 & 1 \\
\hline Ucrania & & 1 & & 1 \\
\hline Venezuela & & 20 & 1 & 21 \\
\hline
\end{tabular}

Fuente: elaboración propia a partir de los datos de Scopus. 
Ilustración 6. Países en los cuales se difunden los artículos de autores colombianos en ciencias económicas (enero de 1974 - junio de 2014)

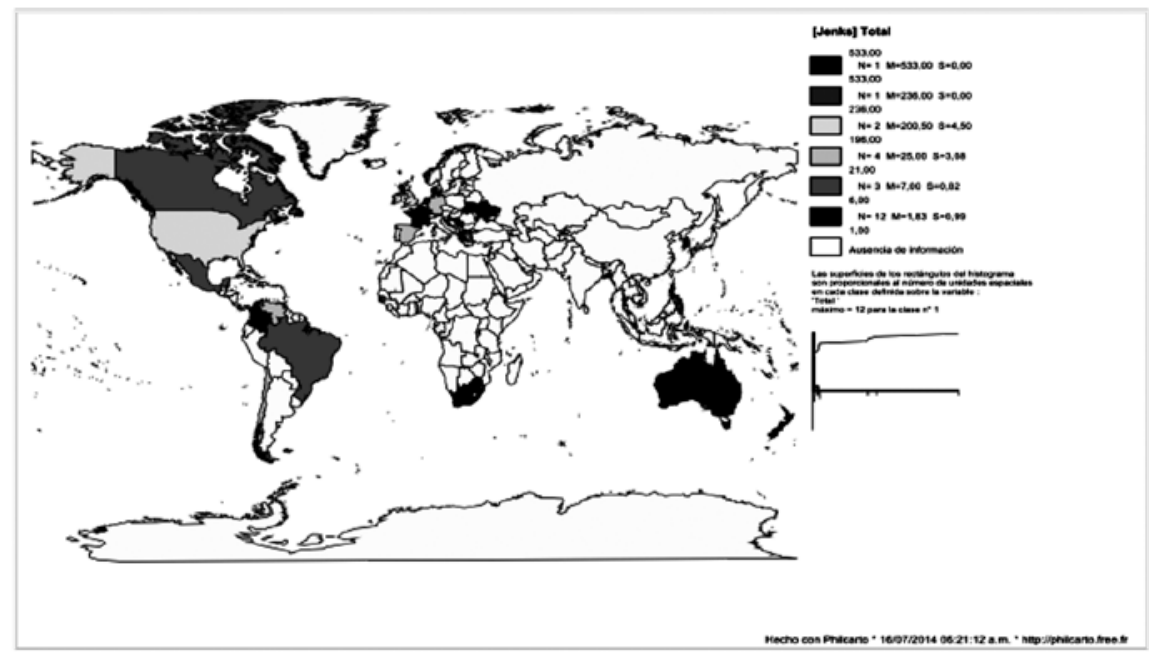

Fuente: elaboración propia a partir de Scopus, empleando Philcato.

Tabla 10. Países en los cuales se difunden los artículos de autores colombianos (discriminados por sexo, enero de 1974 - junio de 2014)

\begin{tabular}{|c|c|c|c|c|c|}
\hline País & No hay autoras & $\begin{array}{l}\text { Hay autoras, pero no } \\
\text { son las principales }\end{array}$ & Autora principal & $\begin{array}{l}\text { Al menos una } \\
\text { autora }\end{array}$ & Total \\
\hline Colombia & 351 & 71 & 111 & 182 & 533 \\
\hline Holanda & 142 & 54 & 40 & 94 & 236 \\
\hline Reino Unido & 114 & 41 & 50 & 91 & 205 \\
\hline Estados Unidos & 113 & 44 & 39 & 83 & 196 \\
\hline Alemania & 10 & 6 & 12 & 18 & 28 \\
\hline Chile & 12 & 7 & 9 & 16 & 28 \\
\hline España & 14 & 3 & 6 & 9 & 23 \\
\hline Venezuela & 12 & 4 & 5 & 9 & 21 \\
\hline Canadá & 3 & 1 & 3 & 4 & 7 \\
\hline México & 5 & 1 & 2 & 3 & 8 \\
\hline Brasil & 4 & 1 & 1 & 2 & 6 \\
\hline Croacia & & & 2 & 2 & 2 \\
\hline Filipinas & 1 & 2 & & 2 & 3 \\
\hline Francia & & & 2 & 2 & 2 \\
\hline Australia & 2 & & 1 & 1 & 3 \\
\hline
\end{tabular}




\begin{tabular}{|l|c|c|c|c|c|}
\hline \multicolumn{1}{|c|}{ País } & No hay autoras & $\begin{array}{c}\text { Hay autoras, pero no } \\
\text { son las principales }\end{array}$ & Autora principal & $\begin{array}{c}\text { Al menos una } \\
\text { autora }\end{array}$ & Total \\
\hline Corea del Sur & 1 & & 1 & 1 & 2 \\
\hline Grecia & & 1 & & 1 & 1 \\
\hline Nueva Zelanda & & 1 & 1 & 1 & 1 \\
\hline Serbia & & & & 2 & 4 \\
\hline Singapur & 2 & 2 & 1 & 0 & 1 \\
\hline Sudáfrica & 1 & & & & 1 \\
\hline Dinamarca & 1 & & & & 1 \\
\hline Ucrania & & & & & 1 \\
\hline
\end{tabular}

Fuente: elaboración propia a partir de los datos de Scopus.

\section{Análisis de citaciones e indicadores}

En este apartado se realiza un análisis de las citaciones y los principales indicadores asociados.

\subsection{Análisis de citaciones}

De los 1.313 trabajos elaborados en el período mencionado, 772 documentos no habían sido citados aún; es decir, el 58,80\% de los documentos producidos entre enero de 1974 y junio de 2014 no habían tenido ninguna repercusión en la producción académica posterior. De otra parte, el número total de citas recibidas durante el período correspondió a 4.115 , en otras palabras, 3,13 citas en promedio por documento realizado y 7,6 citas en promedio por documento realmente citado. Como se aprecia la Ilustración 6, la mayor parte de los documentos elaborados desde 1974 recibe entre 1 y 8 citas, concentrando el 32,29\% de los trabajos y el $27,4 \%$ de las citas recibidas. Este hecho contrasta con el hecho de que 3 documentos concentren el $7,04 \%$ de las citas (Tabla 11).

Ahora bien, de acuerdo con la Tabla 12, los artículos tienen mayor probabilidad de impactar la producción académica posterior si el autor principal es un hombre, pero cuenta con co-autoras. En el caso de autores hombres sin co-autoras, los artículos publicados en revistas multidisciplinarias son menos ci- tados (65,9\%). Los artículos con coautores hombres y mujeres publicados en revistas del área de BMA fueron citados con una frecuencia de 53,5\%. Los trabajos cuyo autor principal es una mujer fueron citados con mayor dificultad si aparecían en revistas multidisciplinarias. La mayor probabilidad de citación en este último caso ocurre en revistas de EEF.

\section{2. Índices $H, G, R$, i10 y $A$}

Aunque tradicionalmente, sólo se calcula el índice $\mathrm{H}$ (Hirsch, 2005, p. 16569) ${ }^{9}$, recientemente se han empleado otros indicadores (Lozano \& Rodríguez, 2012; Arencibia \& Carvajal, 2008) como el G (Egghe, 2006, p. 133) ${ }^{10}$, el i10 ${ }^{11}$, el R (Jin, Liang, Rous-

9 El concepto del índice h aparece en el año 2005 con el trabajo de Hirsh. Su cálculo corresponde a "(...) ordenar los trabajos de un autor de forma decreciente en virtud de las citas recibidas por cada trabajo. En el momento en el que el rango (posición en la lista) supera o iguala al valor de la cita, ahí tenemos nuestro índice h. esto significa que el autor tiene $\mathrm{h}$ trabajos con al menos h citas" (Grupo Scimago, 2007, p. 47).

10 Para el cálculo del Índice G, se listan los artículos de un autor en orden descendente de acuerdo con el número de citas recibidas por cada uno de ellos. Posteriormente, en una segunda columna se calcula el valor del cuadrado del digito que refleja la posición del artículo en la lista (g2). Y en una tercera se refleja el número de citas acumuladas $(\mathrm{H})$. El índice g lo determina la posición del último artículo en el que el valor del cuadrado de esa posición es inferior al número de citas acumuladas (López \& de Pablos, 2013, p. 136; Arencibia \& Carvajal, 2008, p. 3).

11 El índice i10 corresponde al número de publicaciones que han sido citadas al menos 10 veces. 
Ilustración 7. Número de citas vs número de artículos

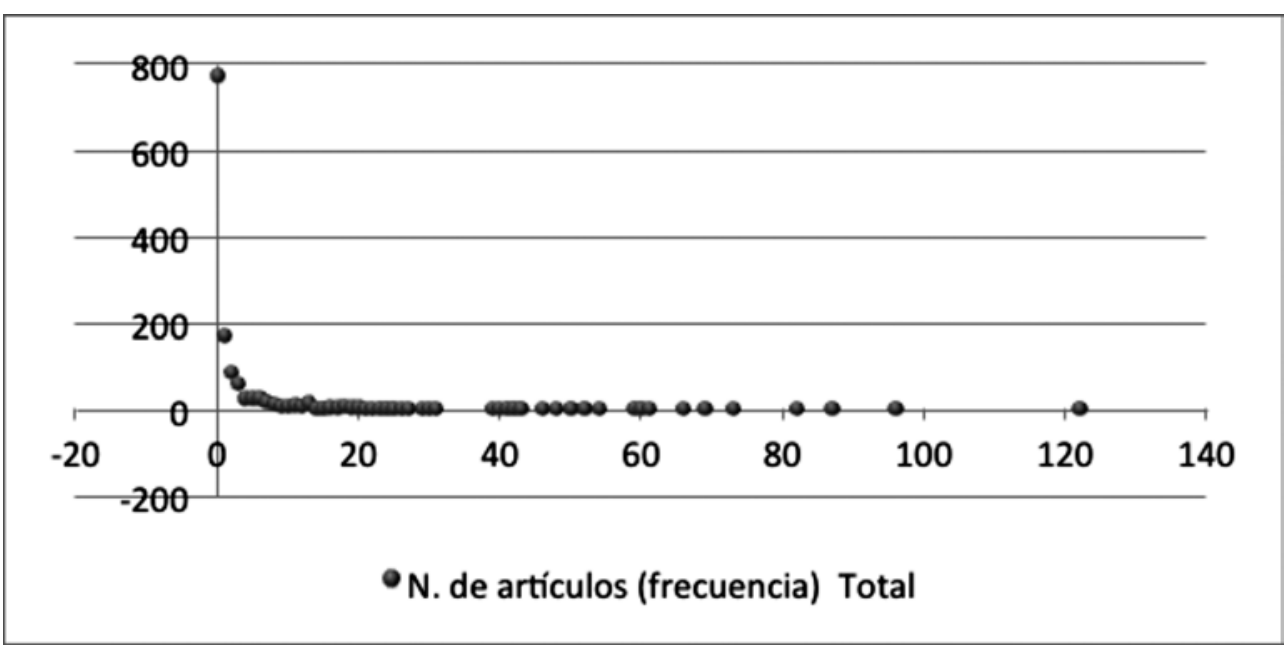

Fuente: elaboración propia a partir de los datos de Scopus.

Tabla 11. Documentos con mayor número de citas

\begin{tabular}{|l|l|c|l|l|l|}
\hline \multicolumn{1}{|c|}{ Autor } & \multicolumn{1}{|c|}{ Título } & \multicolumn{1}{c|}{$\begin{array}{c}\text { Año de } \\
\text { publicación }\end{array}$} & \multicolumn{1}{c|}{ Fuente } & $\begin{array}{c}\text { Número } \\
\text { de citas }\end{array}$ & \multicolumn{1}{c|}{$\begin{array}{c}\text { Tipo de } \\
\text { documento }\end{array}$} \\
\hline $\begin{array}{l}\text { Cardenas J.C., Stranlund J., } \\
\text { Willis C. }\end{array}$ & $\begin{array}{l}\text { Local environmental control and insti- } \\
\text { tutional crowding-out }\end{array}$ & 2000 & World Development & 122 & Artículo \\
\hline Barrios E. & $\begin{array}{l}\text { Soil biota, ecosystem services and } \\
\text { land productivity }\end{array}$ & 2007 & Ecological Economics & 96 & Artículo \\
\hline Esfahani H.S., Ramirez M.T. & $\begin{array}{l}\text { Institutions, infrastructure, and econo- } \\
\text { mic growth }\end{array}$ & 2003 & $\begin{array}{l}\text { Journal of Development } \\
\text { Economics }\end{array}$ & 87 & Artículo \\
\hline Brander J.A. & Chapter 27 Strategic trade policy & 1995 & $\begin{array}{l}\text { Handbook of Internatio- } \\
\text { nal Economics }\end{array}$ & 82 & Capítulo de libro \\
\hline $\begin{array}{l}\text { Bento A.M., Cropper M.L., Mo- } \\
\text { barak A.M., Vinha K. }\end{array}$ & $\begin{array}{l}\text { The effects of Urban spatial structure } \\
\text { on travel demand in the United States }\end{array}$ & 2005 & $\begin{array}{l}\text { Review of Economics } \\
\text { and Statistics }\end{array}$ & 82 & Artículo \\
\hline
\end{tabular}

Fuente: elaboración propia a partir de los datos de Scopus. 
Tabla 12. Documentos citados por sexo en la autoría

\begin{tabular}{|l|c|c|c|c|c|}
\hline & No & Si & Total & No (\%) & Si (\%) \\
\hline Sin autoras & 482 & 306 & 788 & $61,2 \%$ & $38,8 \%$ \\
\hline Ambas & 54 & 28 & 82 & $65,9 \%$ & $34,1 \%$ \\
\hline BMA & 127 & 89 & 216 & $58,8 \%$ & $41,2 \%$ \\
\hline EEF & 301 & 189 & 490 & $61,4 \%$ & $38,6 \%$ \\
\hline Hay autoras, pero no son principales & 126 & 113 & 239 & $52,7 \%$ & $47,3 \%$ \\
\hline Ambas & 23 & 13 & 36 & $63,9 \%$ & $36,1 \%$ \\
\hline BMA & 40 & 46 & 86 & $46,5 \%$ & $53,5 \%$ \\
\hline EEF & 63 & 54 & 117 & $53,8 \%$ & $46,2 \%$ \\
\hline La autora es principal & 164 & 122 & 286 & $57,3 \%$ & $42,7 \%$ \\
\hline Ambas & 21 & 6 & 27 & $77,8 \%$ & $22,2 \%$ \\
\hline BMA & 68 & 40 & 108 & $63,0 \%$ & $37,0 \%$ \\
\hline EEF & 75 & 76 & 151 & $49,7 \%$ & $50,3 \%$ \\
\hline Total & $\mathbf{7 7 2}$ & $\mathbf{5 4 1}$ & $\mathbf{1 3 1 3}$ & $\mathbf{5 8 , 8} \%$ & $\mathbf{4 1 , 2} \%$ \\
\hline
\end{tabular}

Fuente: elaboración propia a partir de los datos de Scopus.

seau \& Egghe, 2009, p. 236) ${ }^{12}$ y el A (Jin, Liang, Rousseau \& Egghe, 2009, p. 237) ${ }^{13}$. El análisis de citas realizado anteriormente permite calcular cada uno de ellos (Tabla 13).

Tabla 13. Índices bibliográficos

\begin{tabular}{|l|c|c|c|c|c|}
\hline & $\begin{array}{c}\text { Índice } \\
\text { H }\end{array}$ & $\begin{array}{c}\text { Índice } \\
\mathbf{R}\end{array}$ & $\begin{array}{c}\text { Índice } \\
\mathbf{G}\end{array}$ & $\mathbf{I 1 0}$ & $\begin{array}{c}\text { Índice } \\
\mathbf{A}\end{array}$ \\
\hline Total cienciaseconómicas & 8 & 20,6 & 44 & 111 & 53 \\
\hline Ambas disciplinas & 3 & 5,84 & 12 & 5 & 11,3 \\
\hline BMA & 6 & 11,7 & 26 & 29 & 22,7 \\
\hline EEF & 8 & 15,4 & 40 & 77 & 29,8 \\
\hline
\end{tabular}

Fuente: elaboración propia a partir de los datos de Scopus.

12 El índice R (Jin, Liang, Rousseau \& Egghe, 2009, p. 236) corresponde a la raíz cuadrada de la sumatoria de citas recibidas por los artículos que hacen parte del índice $\mathrm{H}$.

13 El índice A (Jin, Liang, Rousseau \& Egghe, 2009, p. 237) corresponde a la sumatoria de citas recibidas por los artículos que hacen parte del índice $\mathrm{H}$ dividida entre el índice $\mathrm{H}$.
Considerando los resultados de los índices anteriores se puede deducir que existen diferencias significativas en el impacto de la producción académica de autores con filiación institucional colombiana en las áreas de Economía (Economics, Econometrics and Finance - EEF) y Administración (Business, Management and Accounting - BMA), ya que en todos los casos los indicadores de BMA son más bajos que los de EEF. A su vez, los valores de las revistas que incluyen ambas disciplinas son significativamente menores que los de BMA.

Ahora bien, en la Tabla 14, se muestran los mismos indicadores, pero haciendo la distinción del sexo de los autores. Los índices H, R y A de los autores masculinos sin co-autoría femenina son mayores tanto en el agregado y como segmentando por área de la publicación. A su vez estos indicadores son mayores para los artículos cuyo autor principal es una mujer, en comparación con los trabajos en los cuales hay participación femenina, pero el autor principal es un hombre. 
El indicador I10 es superior para los trabajos en los cuales no participan mujeres, pero a diferencia de los indicadores anteriores, los trabajos publicados en co-autoría con mujeres y cuyo autor principal es hombre tienen un I10 más grande que los artículos en los cuales el autor principal es mujer.

Tabla 14. Índices bibliográficos según sexo en la autoría

\begin{tabular}{|l|c|c|c|c|}
\hline & Índice H & Índice R & I10 & Índice A \\
\hline Sin autoras & 26 & 37,53 & 38 & 54,19 \\
\hline Ambas & 7 & 7,21 & 3 & 7,42 \\
\hline BMA & 11 & 15,03 & 20 & 20,55 \\
\hline EEF & 14 & 24,61 & 36 & 43,29 \\
\hline $\begin{array}{l}\text { Hay autoras, pero no son } \\
\text { principales }\end{array}$ & 8 & 14,52 & 27 & 26,38 \\
\hline Ambas & 4 & 4 & 3 & 4 \\
\hline BMA & 8 & 9,48 & 12 & 11,25 \\
\hline EEF & 7 & 10,25 & 19 & 15 \\
\hline La autora es principal & 9 & 17,21 & 23 & 32,89 \\
\hline Ambas & 3 & 2,45 & 0 & 2 \\
\hline BMA & 9 & 10,15 & 11 & 11,44 \\
\hline EEF & 9 & 13 & 19 & 18,78 \\
\hline
\end{tabular}

Fuente: elaboración propia a partir de los datos de Scopus.

\section{Indicadores de género}

En este apartado se presentan los indicadores de género que se encuentran en la Tabla 17 (Anexos), aplicados al número de autores (Tabla 15). Como ya se había visto en la sección de autores, el 24\% son mujeres y el $76 \%$ son hombres. La mayor parte de autores (hombres o mujeres) publicaron trabajos en el área de EEF. En las publicaciones multidiscuiplinares y de BMA la distribución vertical de las mujeres es superior a la de los hombres.

El índice de feminidad (IF) indica que por cada 100 hombres hay 32 mujeres en el agregado. El IF más alto se presenta en las publicaciones de BMA (37). Por su parte el índice de masculinidad (IM), es decir, número de hombres por cada 100 mujeres, fue de 317 en el agregado. El IM más alto se presentó en las publicaciones de EEF.

Al establecer la relación entre el IM y el IF, se puede indicar que dentro de un grupo de 100 personas, por cada mujer que publique en una revista multidisciplinar lo harán aproximadamente 9 hombres, en el área BMA por cada mujer habrá aproximadamente 7 autores hombres, y en el EEF esta relación será 1 a 13.

El indicador de segregación indica que considerando la proporción de autores y autoras, en relación con la población del mismo sexo, no hay señales de segregación en alguno de los tipos de publicación: multidisciplinar, BMA y EEF. Sin embargo, el índice de disimilitud establece que el $6,61 \%$ de los autores hombres deberían publicar en otra área para hubiera paridad completa.

En cuanto al índice de contribución al sexismo se aprecia que todas las áreas de publicación favorecen la masculinización, sin embargo, EEF es la que más la favorece al tener un valor superior a la unidad. De forma consistente, con un mayor IF, BMA es la que menos favorece la masculinización.

Finalmente, el indicador de interacción establece que un autor que publique en alguna de las tres áreas de ciencias económicas, tiene que en promedio por cada 10 autores, este interactuará con aproximadamente 8 hombres y 2 mujeres.

\section{Consideraciones finales}

El documento presentado muestra la combinación entre un análisis bibliométrico y de género. Aunque el debate sobre el papel de la mujer en el mundo académico se ha centrado en el estudio de los diversos fenómenos que podrían ser la causa de la brecha de género, son pocos los trabajos que busquen cuantificarla en la esfera de la producción y publicación académica. 
Tabla 15. Indicadores de género aplicados al sexo de los autores

\begin{tabular}{|l|c|c|c|c|}
\hline \multicolumn{1}{|c|}{ Variable } & Ambas & BMA & EEF & Total \\
\hline Hombres autores & 298 & 827 & 1274 & 2399 \\
\hline Mujeres autoras & 100 & 305 & 352 & 757 \\
\hline Total autores & 398 & 1132 & 1626 & 3156 \\
\hline Distribución horizontal hombres & $9,44 \%$ & $26,20 \%$ & $40,37 \%$ & $76,01 \%$ \\
\hline Distribución horizontal mujeres & $3,17 \%$ & $9,66 \%$ & $11,15 \%$ & $23,99 \%$ \\
\hline Distribución vertical hombres & $12,42 \%$ & $34,47 \%$ & $53,11 \%$ & $100,00 \%$ \\
\hline Distribución vertical mujeres & $13,21 \%$ & $40,29 \%$ & $46,50 \%$ & $100,00 \%$ \\
\hline Índice de feminidad (IF) & 34 & 37 & 28 & 32 \\
\hline Índice de masculinidad (IM) & 298 & 271 & 362 & 317 \\
\hline IM /IF & 8,88 & 7,35 & 13,10 & 10,04 \\
\hline Indicador de segregación & & & & 0,0660612 \\
\hline Índice de disimilaridad de Duncan & & & & $6,61 \%$ \\
\hline Índice de contribución al sexismo & 0,95619388 & 0,88631463 & 1,08987 & \\
\hline Índice de interacción & & & & 7,57589284 \\
\hline
\end{tabular}

Fuente: elaboración propia a partir de los datos de Scopus.

El análisis realizado permitió identificar que se presenta un fenómeno de masculinización de la producción académica de autores colombianos, en ciencias económicas. Si bien el número de autoras en Economía y Administración ha ido en aumento, su participación aún es limitada, pero debe aclararse que la incorporación de mujeres en la producción académica se ha incrementado más que la participación de los hombres.

El área con mayor tasa de autoras principales fue BMA con $26,3 \%$ y es precisamente el área en la cual hay mayor paridad en la autoría de artículos, $50,78 \%$ de los trabajos son escritos por hombres sin la participación de mujeres.

En términos absolutos, la revista en la cual más artículos con participación de mujeres han sido publicados durante el período, fue Cuadernos de Administración (98 trabajos, 42,9\%), seguida de la revista Innovar (87 trabajos, 42,5\%). Frente a las fuentes, se identificó también que la mayor parte de las revistas que concentran las publicaciones de mujeres son colombianas. Hubo 148 fuentes que habían publicado solamente trabajos de autores hombres en ciencias económicas. Los países con mayor publicación de artículos con participación de mujeres fueron Colombia (182), Holanda (94), Reino Unido (91) y Estados Unidos (83).

En los 1.313 trabajos analizados, participaron 3.156 autores, de los cuales sólo 756 eran mujeres, es decir el 24\%. En las publicaciones multidisciplinarias la participación de las mujeres fue de 25,1\%, en BMA 26,9\% y en EEF 21,6\%.

Adicionalmente, se identificó que los artículos tienen mayor probabilidad de impactar la producción académica posterior -es decir, al menos llegar a ser citado una vez-, si el autor principal es un hombre, pero cuenta con co-autoras. No obstante, los mejores indicadores en cuanto a reiteración de las cita- 
ciones (índices $\mathrm{H}, \mathrm{R}$ y A) los obtienen los artículos escritos por hombres sin co-autoría femenina.

Los indicadores de género calculados con respecto al número de autores, reiteran la brecha de género y la masculinización en la publicación científica de ciencias económicas, siendo EEF la que más contribuye a la masculinización, durante el período analizado. Sin embargo, los indicadores no evidencian que haya una dinámica de segregación en las tres áreas analizadas.

Para los futuros trabajos a realizar queda la pregunta latente de identificar ¿cuáles son los factores o las razones que explican el fenómeno de masculinización de la producción académica de autores colombianos en ciencias económicas?

\section{Referencias}

Abramo, G., Cicero, T. \& D'Angelo, C. (2014). Are the authors of highly cited articles also the most productive ones? Journal of Informetrics, 8(1): 89-97.

Abramo, G., D’Angelo, C. \& Murgia, G. (2013). Gender differences in research collaboration. Journal of Informetrics, 7(4): 811-822.

Albert, C. (2000). Higher education demand in Spain; the influence of labour market signals and family background. Higher Education, 40(2): 147-162.

Albert, C., González, C. \& Mora, J. (2011). Análisis de la evolución y caracterización de la demanda de educación universitaria en Colombia (Borradores de Economía y Finanzas, 28). Cali: ICESI. Disponible en: http://bibliotecadigital.icesi.edu.co/ biblioteca_digital/bitstream/10906/67533/1/analisis_evolucion_caracterizacion.pdf

Ale Ebrahim, N., Salehi, H., Embi, M., Habibi, F., Gholizadeh, H. \& Motahar, S. (2014). Visibility and Citation Impact. International Education Studies, 7(4): 120-125.

Aleixandre, R., González, G., Alonso, A., Castellano, M. \& Valderrama, J. (2007). Valoración de la paridad en la autoría de los artículos publicados en la Revista Enfermedades Infecciosas y Microbiología Clínica durante el quinquenio 2001-2005. Enfermedades Infecciosas y Microbiología Clínica, 25(10): 619-626.

Alonso, A., Aleixandre, R., Vidal, A., Anguita, M., Chorro, F., Bolaños, M., Castelló, L., Navarro, C. \& Valderrama, J. (2014). Publicaciones derivadas de las comunicaciones a los congresos anuales de la Sociedad Española de Cardiología. Revista Española de Cardiología, 67(1): 15-21.

Amodio, P. \& Brugnano, L. (2014). Recent advances in bibliometric indexes and the PaperRank problem. Journal of Computational and Applied Mathematics, 267: 182-194.

Araújo, J. \& Arencibia, R. (2002). Informetría, bibliometría y cienciometría: aspectos teórico-prácticos. ACIMED, 10 (4): 5-6.
Arencibia, R. \& Carvajal, R. (2008). Los índices H, G y R: su uso para identificar autores líderes en el área de la Comunicación durante el período 2001-2006. ACIMED, 17(4).

Arias, E., Velasco, J. \& Novo, M. (2016). Análisis bibliométrico sobre la investigación en violencia de género. Fundamentos y nuevas tendencias. Disponible en: https://www.researchgate.net/ profile/Esther_Arias/publication/296485748_ANALISIS_BIBLIOMETRICO_SOBRE_LA_INVESTIGACION_EN_VIOLENCIA_DE_GENERO_FUNDAMENTOS_Y_NUEVAS_TENDENCIAS_BIBLIOMETRIC_ANALYSIS_ON_INTIMATE_PARTNER_VIOLENCE_RESEARCH_BASIS_AND_NEW_TRENDS/ links/56d5dd7108aee1aa5f730caa.pdf

Arias, A. (2015). Nuevas aproximaciones metodológicas al estudio de la colaboración en la ciencia a través de las publicaciones científicas. [Tesis doctoral] Salamanca: Universidad de Salamanca. Disponible en: http://hdl.handle. net/10366/127969

Armfield, N., Edirippulige, S., Caffery, L., Bradford, N., Grey, J. \& Smith, A. (2014). Telemedicine - A bibliometric and content analysis of 17,932 publication records. International Journal of Medical Informatics, In Press, Corrected Proof.

Bailyn, L. (2003). Academic Careers and Gender Equity: Lessons Learned from MIT. Gender, Work and Organisation, 10(2): 137-153.

Benoit, M. (2015). Qui fait quoi? Analyse des libellés de contribution dans les articles savants [Tesis de Maestría]. Montreal: Université de Montreal. Disponible en: https://papyrus.bib. umontreal.ca/xmlui/handle/1866/12541

Bonilla,V., López de Méndez, A., Cintrón, M., Ramírez, S. \& Román, R. (2005). Feminización de la matrícula de Educación Superior en Puerto Rico. Disponible en: http://cie.uprrp.edu/ cuaderno/ediciones/20/pdf/c20art7.pdf

Bouyssou, D. \& Marchant, T. (2014). An axiomatic approach to bibliometric rankings and indices. Journal of Informetrics, 8(3): 449-477.

Brooks, C., Fenton, E. \& Walker, J. (2014). Gender and the evaluation of research. Research Policy, 43(6): 990-1001.

Brones, F., Monteiro de Carvalho, M. \& de Senzi Zancul, E. (2014). Ecodesign in project management: a missing link for the integration of sustainability in product development? Journal of Cleaner Production, 80(1): 106-118.

Brzezinski, M. (2014). Power Laws in Citation Distributions: Evidence from Scopus Disponible en: http://ssrn.com/abstract=2397685 or http://dx.doi.org/10.2139/ssrn.2397685

Bustos, O. \& Blázquez, N. (2003). Qué dicen las académicas acerca de la UNAM. México: UNAM.

Buquet, A., Cooper, J. \& Rodríguez, H. (2010). Sistema de indicadores para la equidad de género en instituciones de educación superior. México: UNAM. Disponible en: http:// www.pueg.unam.mx/images/stories/Equidad/Investigacion/ sist.\%20de\%20indicadores.pdf

Buquet, A., Cooper, J., Rodríguez, H. \& Botello, L. (2006). Presencia de mujeres y hombres en la UNAM: una radiografía. México: UNAM.

Buquet, A., Hernández, A. \& Jiménez, V. (2011). Diagnóstico de la situación de mujeres y hombres por dependencia. Instituto de Matemáticas de la UNAM. México D.F.: UNAM.

Caputo, C., Vargas, D. \& Requena, J. (2016). Desvanecimiento de la brecha de género en la universidad venezolana. Interciencia, 41(3): 154-161. 
Correa, M. (2003). Los géneros en la educación superior en Colombia. Santiago de Chile: Instituto Internacional para la Educación Superior en América Latina y el Caribe.

Costa, P. R. da. (2015). Inovação no ensino e aprendizagem em finanças: análise da literatura entre 2005 e 2015. [Tesis de maestría]. São Paulo: FECAP.

Daza, S. (2010). Las mujeres en el SNCTI. Balance de una década en condiciones diferentes. En OCyT, Indicadores de Ciencia y Tecnología (pp. 279-316). Bogotá: OCyT.

Daza, S. \& Pérez, T. (2008). Contando mujeres. Una reflexión sobre los indicadores de género y ciencia en Colombia. Revista de Antropología y sociología Virajes, 10: 29-51.

De Oliveira, M., Mazer, S., Guillaumon, M. \& Fernández, E. (2014). Análisis de la producción científica en Brasil sobre dificultades de aprendizaje: una revisión bibliométrica. Aula Abierta, 42(1): 31-38.

De Pablos, L. \& Gil, M. (2011). Las políticas de educación desde la perspectiva de género. Presupuesto y Gasto Público, 64: 179-208.

Delgado, E. (2016). ¿Evaluar la investigación con Google Scholar? Yes we can. Ponencia presentada en el V Seminario EC3, celebrado en Granada el 12 de junio de 2015. Disponible en: http://hdl.handle.net/10481/41069

Egghe, L. (2006). Theory and practise of the g-index. En: Scientometrics, 69(1): 131-152.

Eraso, L. (2016). La mujer en la medicina colombiana. Revista Medicina, 38(1): 73-81.

Escolano, E. (2006). Discriminación en un medio meritocrático: las profesoras en la Universidad española. Revista Mexicana de Sociología, 2, 231-263. Disponible en: http://www.revistas. unam.mx/index.php/rms/article/view/6055

Escolano, E. (2009). El poder como asignatura pendiente de las académicas en las universidades españolas. En: Chávez, M. A., Chávez, M.R, Ramírez, E., Cruz, M. \& Cervantes, G. Género y trabajo en las universidades (pp. 83-127). Guadalajara: Universidad de Guadalajara.

Estébañez, M. (2007). Género e investigación científica en las universidades latinoamericanas. Educación superior y sociedad, 1 (1): 1-26

Galvéz, J. (2009). La mujer a través del telescopio. Disponible en http://www.oei.es/cienciayuniversidad/spip.php?article109

García, P. (2009). Las académicas entre la materialidad política y la subjetividad. En: Chávez, M.A., Chávez, M.R, Ramírez, E., Cruz, M. \& Cervantes, G. Género y trabajo en las universidades (pp. 31-48). Guadalajara: Universidad de Guadalajara.

García, P. (2004). Mujeres académicas: el caso de una universidad estatal mexicana. México: Plaza y Valdés. Universidad de Guadalajara.

García, P. (1992). Notas sobre la participación de la mujer académica en la Universidad de Guadalajara. Revista Tiempo de Ciencia, 28: 33-36.

González, B., Guerrero, V. \& Moya, F. (2009). The SJR indicator: A new indicator of journals' scientific prestige. Tech. Rep. arxiv:abs/0912.4141 [cs.DL]. Consultado el 10 de junio de 2010 en http://arxiv.org/ftp/arxiv/papers/0912/0912.4141.pdf.

Gregorio, O., Méndez, C. \& Peralta, M. (2015). Acercamiento bibliométrico a las revistas científicas colombianas de ciencias sociales: comparación y nuevas miradas hacia la evaluación y categorización a partir de ISI WoS y Scielo Citation Index. IV Jornadas de Intercambio y Reflexión acerca de la Investigación en Bibliotecología. Disponible en: http://hdl.handle. net/10915/52479
Grupo Scimago (2007). "El índice h de Hirsch: su aplicación a algunos de los científicos españoles más destacados". El profesional de la información, 16(1): 47-49.

Filippo, D. \& Fernández, M. (2002). Bibliometría: importancia de los indicadores bibliométricos. El estado de la ciencia: principales indicadores deficiencia y tecnología iberoamericanos/interamericanos. Disponible em: http://www.ricyt.org/ component/docman/doc_view/113-bibliometria-importanciade-los-indicadores-bibliometricos?Itemid $=2$

Hagen, N. (2014). Reversing the byline hierarchy: the effect of equalizing bias on the accreditation of primary, secondary and senior authors. Journal of Informetrics, 8(3): 618-627.

Hirsch, J. (2005).An index to quantify an individual's scientific research output, Proceedings of the National Academy of Sciences, 102 (46): 16569-16572.

Jatobá, I. \& Gomes, J. (2012). Cartografia digital: o software philcarto no ensino da geocartografia. Revista Metáfora Educacional, 12: 49-65.

Jin, B., Liang L., Rousseau, R. \& Egghe, L. (2007). The R- and AR-indices: Complementing the h- index. Chinese Science Bulletin, 52(6): 855-863.

Joyce, C., Kelly, J. \& Sugrue, C. (2014). A bibliometric analysis of the 100 most influential papers in burns. Burns, 40(1): 30-37.

Kretschmer, H. \& Aguillo, I. (2005). New indicators for gender studies in Web networks. Information Processing \& Management, 41(6): 1481-1494.

Koc, E. \& Boz, H. (2014). Triangulation in tourism research: A bibliometric study of top three tourism journals. Tourism Management Perspectives, 12: 9-14.

Lemarchand, G. (ed.) (2010). Sistemas Nacionales de ciencia, tecnología e innovación en América Latina y el Caribe. Montevideo: Unesco.

Lis-Gutiérrez, J-P. (2012). Análisis de los grupos de investigación colombianos en ciencias económicas desde una perspectiva de género. Revista de la Facultad de Ciencias Económicas, XX (2): 143-164

López, M. \& de Pablos, J. (2013). El" índice h" en las estrategias de visibilidad, posicionamiento y medición de impacto de artículos y revistas de investigación. Investigar la Comunicación hoy. Revisión de políticas científicas y aportaciones metodológicas: Simposio Internacional sobre Política Científica en Comunicación, 133-150.

Lozano, I. \& Rodríguez, Y. (2012). Análisis de los índice H, G Y R en el sector agropecuario cubano a través de Scopus, 2005-2009. Anales de Documentación, 15(1): 1-17.

Lozano, I., Iglesias, M. \& Martínez, M. (2016). Un estudio cualitativo sobre los diferenciales de género en la educación superior: percepciones de las académicas en contextos masculinizados. La manzana de la discordia, 11 (1): 41-54.

López, J., Basora, J., Orozco, D. \& Bellón, J. (2014a). Mapa bibliométrico de la investigación realizada en atención primaria en España durante el periodo 2008-2012. Atención Primaria, In Press, Corrected Proof.

López, S., Svider, P. Misra, P., Bhagat, N., Langer, P. \& Eloy, J. (2014b). Gender differences in promotion and scholarly impact: an analysis of 1460 academic ophthalmologists. Journal of Surgical Education, In Press, Corrected Proof.

Lozano, I. \& Rodríguez, Y. (2012). Análisis de los índice H, G Y R en el sector agropecuario cubano a través de Scopus, 2005-2009. Anales de Documentación, 15(1): 1-17. 
Machado, C., Saraiva de Souza, M., dos Santos Parisotto, I. \& Palmisano, A. (2016). As Leis da Bibliometria em Diferentes Bases de Dados Científicos. Ciencias da Administraçao, 18(44): 111-123.

Martínez, J., Ríos, J., Meroño, A., Martínez, J. \& del-Baño, M. (2014). Caracterización de la base intelectual de la fisioterapia a través del análisis de cocitación de documentos. Fisioterapia, 36(4): 167-176.

Melo, H. (2013). Avaliação da Produção Acadêmica da Revista Gestão \& Regionalidade de 2005 a 2012 através de Bibliometria e Sociometria. Gestão e Sociedade, 7 (18). Doi: http:// dx.doi.org/10.21171/ges.v7i18.1899

Observatorio Colombiano de Ciencia y Tecnología OCyT (2009). Indicadores de Ciencia y Tecnología, 2008. Bogotá: Observatorio Colombiano de Ciencia y Tecnología. Disponible en: http://ocyt.org.co/es-es/InformeAnuallndicadores/ArtMID/542/ArticleID/17/Libro-de-Indicadores-de-Ciencia-yTecnolog237a-2008

Ortega, E., Valdivia, P., Olmedilla, A., Martínez, M. \& Villarejo, D. (2015). Estudio bibliométrico del papel de la mujer en las tesis doctorales de ciencias del deporte. Journal of Sport and Health Research, 7(2): 139-148.

Ortiz, E. \& Hidalgo, Y. (2016). Detección de comunidades a partir de redes de coautoría en grafos RDF. Revista Cubana de Información en Ciencias de la Salud, 27(1): 90-99.

Papadópulos, J. \& Radakovich, R. (2006). Educación superior y género en América Latina y el Caribe. En: Informe sobre la Educación Superior en América Latina y el Caribe 2000-2005. La metamorfosis de la educación superior. Caracas: UNESCO, IESALC.

Pérez, T. (2012). Miércoles de ciencia y tecnología. Un Radio. Podcast disponible en http://www.unradio.unal.edu.co/detalle/ cat/un-analisis/article/mujeres-cientificas.html

Portugal, M., Carvalho, J., Ribeiro de Almeida, M. \& Reis, N. (2014). Mergers \& acquisitions research: A bibliometric study of top strategy and international business journals, 1980-2010. Journal of Business Research, In Press.

Pritchard, A. (1969). Statistical bibliography or bibliometrics. Journal of documentation, 25: 348-349.

Ponomariov, B. \& Toivanen, H. (2014). Knowledge flows and bases in emerging economy innovation systems: Brazilian research 2005-2009. Research Policy, 43(3): 588-596.

Rehn, C., \& Kronman, U. (2008). Bibliometric handbook for Karolinska Institutet. Huddinge: Karolinska Institutet.

Requena, J., Vargas, D. \& Caputo, C. (2016). Género en la ciencia venezolana: desvanecimiento de la brecha. Interciencia, 41(3) , 162-170. Disponible en http://www.interciencia. org/v41_03/162.pdf

Restrepo, C. \& Urbizagástegui, R. (2016). Métrica de la literatura sobre los indígenas de México. Encontros Bibli: revista eletrônica de biblioteconomia e ciência da informação, 21(46): 104-120.

Rubio, M. (1999). Bibliometría y ciencias sociales. Clio, 7. Disponible en http://clio.rediris.es/numero007.html. Consultado el 20 de septiembre de 2010.

SCImago (2016). SJR - SCImago Journal \& Country Rank. Disponible en: http://www.scimagojr.com

Scopus (2016). Content overview. Disponible en: http://www. elsevier.com/online-tools/scopus/content-overview

Schreiber, M. (2014). Examples for counterintuitive behavior of the new citation-rank indicator P100 for bibliometric evaluations. Journal of Informetrics, 8(3): 738-748.
Si Niu, X. (2014). International scientific collaboration between Australia and China: A mixed-methodology for investigating the social processes and its implications for national innovation systems. Technological Forecasting and Social Change, 85: 58-68.

Silva Andrade, L., de Paiva, A., de Castro Alcântara, V. \& Brito, M. (2016). Desvelando o Campo da Estratégia como Prática e suas Relações, Iberoamerican Journal of Strategic Management, 15(1).

Sos Peña, R. (2015). La influencia de las primeras psicólogas norteamericanas en la historia de la psicología. Revista de Historia de la Psicología, 36(2): 31-46.

Tomei, K., Nahass, M., Husain, Q., Agarwal, N., Patel, S., Svider, P., Eloy, J. \& Liu, J. (2014). A gender-based comparison of academic rank and scholarly productivity in academic neurological surgery. Journal of Clinical Neuroscience, 21(7): 1102-1105.

Tovar, P. (2002). Género y ciencia en Colombia: Algunos indicadores. En: Colombia Ciencia y Tecnología, 20 (2). Bogotá: Colciencias.

Tovar, P. (2004). Indicadores nacionales de género, ciencia y tecnología. En: Memorias seminario: Las mujeres colombianas en el sistema de ciencia y tecnología: obstáculos y logros. Bogotá: ICANH. Abril 19-21 de 2006.

Tovar, P. (2005). La percepción que tienen los colombianos sobre la ciencia y la tecnología: la importancia de tener una perspectiva de género. En: Aguirre, J. (ed.). La percepción que tienen los colombianos sobre la ciencia y la tecnología. Bogotá: Colciencias.

Túñez, M. \& de Pablos, J. (2013). El "índice h" en las estrategias de visibilidad, posicionamiento y medición de impacto de artículos y revistas de investigación. En: Pacheco, M., Rueda, M., Mariño, V. \& González, T. (coords.). 2º Congreso Nacional sobre Metodología de la Investigación en Comunicación ( $p$. 133-150). Valladolid: Facultad de Ciencias Sociales, Jurídicas y de la Comunicación.

UNESCO (2009). S\&T World Data Fact Sheet. Montreal: Unesco. Valcárcel de Laiglesia, M., Alfonso, F., Miró, O., Casademont, J., Burbano Santos, P., Burillo, G., Fernández, C. \& Martín, F. (2014). Characteristics and Longevity of Electronic Citations in Four Leading Biomedical Journals in Spain. Revista Española de Cardiología (English Edition), In Press.

Veiga, D., Conforto, G., Malheiros, M., Carneiro, M., Cohen, M. \& da Silveira, C. (2014). Níveis de evidência da cirurgia de joelho em periódicos nacionais. Revista Brasileira de Ortopedia, 49(1): 13-16.

Vieira, E., Cabral, J. \& Gomes, J. (2014). How good is a model based on bibliometric indicators in predicting the final decisions made by peers? Journal of Informetrics, 8(2): 390-405.

Yu, C., Davis, C. \& Dijkema, G. (2014). Understanding the Evolution of Industrial Symbiosis Research (April 2014). Journal of Industrial Ecology, 18(2): 280-293.

Weiss, D., Kovshilovskaya, B. \& Breyer, B. (2012). Gender Trends of Urology Manuscript Authors in the United States: A 35Year Progression. The Journal of Urology, 187(1): 253-258.

Zapata, M. (2010). La equidad de género en las universidades alemanas. En: Mingo, A. (ed.) Desasosiegos. Relaciones de género en la educación (pp. 109-150). México D.F.: Instituto de Investigaciones sobre la Universidad y la Educación, UNAM. 


\section{ANEXOS}

Tabla 16. Citas recibidas por los documentos sobre desarrollo territorial (1975-2010)

\begin{tabular}{|c|c|c|c|c|c|c|c|}
\hline $\begin{array}{l}\text { Número } \\
\text { de citas }\end{array}$ & $\begin{array}{l}\text { N. de artículos } \\
\text { (frecuencia) } \\
\text { Ambas }\end{array}$ & $\begin{array}{l}\text { N. de artículos (frecuencia) } \\
\text { Business, Management and } \\
\text { Accounting }\end{array}$ & $\begin{array}{l}\text { N. de artículos (frecuen- } \\
\text { cia) Economics, Econo- } \\
\text { metrics and Finance }\end{array}$ & $\begin{array}{l}\text { N. de artículos } \\
\text { (frecuencia) } \\
\text { Total }\end{array}$ & $\begin{array}{c}\% \\
\text { documentos }\end{array}$ & $\begin{array}{l}\text { Total de } \\
\text { citas }\end{array}$ & $\%$ citas \\
\hline 1 & 25 & 56 & 90 & 171 & $13,02 \%$ & 171 & $4,2 \%$ \\
\hline 2 & 4 & 30 & 53 & 87 & $6,63 \%$ & 174 & $4,2 \%$ \\
\hline 3 & 5 & 24 & 30 & 59 & $4,49 \%$ & 177 & $4,3 \%$ \\
\hline 4 & 2 & 7 & 17 & 26 & $1,98 \%$ & 104 & $2,5 \%$ \\
\hline 5 & 2 & 10 & 14 & 26 & $1,98 \%$ & 130 & $3,2 \%$ \\
\hline 6 & 2 & 9 & 15 & 26 & $1,98 \%$ & 156 & $3,8 \%$ \\
\hline 7 & 2 & 4 & 11 & 17 & $1,29 \%$ & 119 & $2,9 \%$ \\
\hline 8 & & 4 & 8 & 12 & $0,91 \%$ & 96 & $2,3 \%$ \\
\hline 9 & & 2 & 4 & 6 & $0,46 \%$ & 54 & $1,3 \%$ \\
\hline 10 & 1 & 2 & 5 & 8 & $0,61 \%$ & 80 & $1,9 \%$ \\
\hline 11 & & 2 & 7 & 9 & $0,69 \%$ & 99 & $2,4 \%$ \\
\hline 12 & & 2 & 4 & 6 & $0,46 \%$ & 72 & $1,7 \%$ \\
\hline 13 & & 4 & 10 & 14 & $1,07 \%$ & 182 & $4,4 \%$ \\
\hline 14 & & & 3 & 3 & $0,23 \%$ & 42 & $1,0 \%$ \\
\hline 15 & 1 & 2 & & 3 & $0,23 \%$ & 45 & $1,1 \%$ \\
\hline 16 & & 3 & 2 & 5 & $0,38 \%$ & 80 & $1,9 \%$ \\
\hline 17 & & 2 & 2 & 4 & $0,30 \%$ & 68 & $1,7 \%$ \\
\hline 18 & 1 & 1 & 5 & 7 & $0,53 \%$ & 126 & $3,1 \%$ \\
\hline 19 & & 1 & 3 & 4 & $0,30 \%$ & 76 & $1,8 \%$ \\
\hline 20 & & 1 & 4 & 5 & $0,38 \%$ & 100 & $2,4 \%$ \\
\hline 21 & & & 2 & 2 & $0,15 \%$ & 42 & $1,0 \%$ \\
\hline 22 & 1 & & 2 & 3 & $0,23 \%$ & 66 & $1,6 \%$ \\
\hline 23 & & 1 & 2 & 3 & $0,23 \%$ & 69 & $1,7 \%$ \\
\hline 24 & & & 1 & 1 & $0,08 \%$ & 24 & $0,6 \%$ \\
\hline 25 & & & 2 & 2 & $0,15 \%$ & 50 & $1,2 \%$ \\
\hline 26 & & 1 & 1 & 2 & $0,15 \%$ & 52 & $1,3 \%$ \\
\hline 27 & & & 2 & 2 & $0,15 \%$ & 54 & $1,3 \%$ \\
\hline 29 & & 1 & & 1 & $0,08 \%$ & 29 & $0,7 \%$ \\
\hline
\end{tabular}




\begin{tabular}{|c|c|c|c|c|c|c|c|}
\hline $\begin{array}{l}\text { Número } \\
\text { de citas }\end{array}$ & $\begin{array}{l}\text { N. de artículos } \\
\text { (frecuencia) } \\
\text { Ambas }\end{array}$ & $\begin{array}{c}\text { N. de artículos (frecuencia) } \\
\text { Business, Management and } \\
\text { Accounting }\end{array}$ & $\begin{array}{l}\text { N. de artículos (frecuen- } \\
\text { cia) Economics, Econo- } \\
\text { metrics and Finance }\end{array}$ & $\begin{array}{l}\text { N. de artículos } \\
\text { (frecuencia) } \\
\text { Total }\end{array}$ & $\begin{array}{c}\% \\
\text { documentos }\end{array}$ & $\begin{array}{l}\text { Total de } \\
\text { citas }\end{array}$ & $\%$ citas \\
\hline 30 & & & 1 & 1 & $0,08 \%$ & 30 & $0,7 \%$ \\
\hline 31 & & & 1 & 1 & $0,08 \%$ & 31 & $0,8 \%$ \\
\hline 39 & 1 & & 1 & 2 & $0,15 \%$ & 78 & $1,9 \%$ \\
\hline 40 & & 1 & & 1 & $0,08 \%$ & 40 & $1,0 \%$ \\
\hline 41 & & & 2 & 2 & $0,15 \%$ & 82 & $2,0 \%$ \\
\hline 42 & & & 1 & 1 & $0,08 \%$ & 42 & $1,0 \%$ \\
\hline 43 & & & 1 & 1 & $0,08 \%$ & 43 & $1,0 \%$ \\
\hline 46 & & & 1 & 1 & $0,08 \%$ & 46 & $1,1 \%$ \\
\hline 48 & & & 1 & 1 & $0,08 \%$ & 48 & $1,2 \%$ \\
\hline 50 & & & 1 & 1 & $0,08 \%$ & 50 & $1,2 \%$ \\
\hline 52 & & & 2 & 2 & $0,15 \%$ & 104 & $2,5 \%$ \\
\hline 54 & & 1 & & 1 & $0,08 \%$ & 54 & $1,3 \%$ \\
\hline 59 & & & 1 & 1 & $0,08 \%$ & 59 & $1,4 \%$ \\
\hline 60 & & 1 & & 1 & $0,08 \%$ & 60 & $1,5 \%$ \\
\hline 61 & & 1 & & 1 & $0,08 \%$ & 61 & $1,5 \%$ \\
\hline 66 & & & 1 & 1 & $0,08 \%$ & 66 & $1,6 \%$ \\
\hline 69 & & 1 & & 1 & $0,08 \%$ & 69 & $1,7 \%$ \\
\hline 73 & & 1 & 1 & 2 & $0,15 \%$ & 146 & $3,5 \%$ \\
\hline 82 & & & 2 & 2 & $0,15 \%$ & 164 & $4,0 \%$ \\
\hline 87 & & & 1 & 1 & $0,08 \%$ & 87 & $2,1 \%$ \\
\hline 96 & & & 1 & 1 & $0,08 \%$ & 96 & $2,3 \%$ \\
\hline 122 & & & 1 & 1 & $0,08 \%$ & 122 & $3,0 \%$ \\
\hline 0 & 98 & 235 & 439 & 772 & $58,80 \%$ & 0 & $0,0 \%$ \\
\hline Total & 145 & 410 & 758 & 1313 & $100,00 \%$ & 4.115 & $100,0 \%$ \\
\hline
\end{tabular}

Fuente: elaboración propia a partir de los datos de Scopus. 
Tabla 17. Indicadores de gênero calculados

\begin{tabular}{|c|c|c|c|}
\hline Indicador & Explicación & Cálculo & Interpretación \\
\hline $\begin{array}{l}\text { Distribución } \\
\text { horizontal }\end{array}$ & $\begin{array}{l}\text { Participación relativa de mujeres y } \\
\text { hombres en un ambiente i determi- } \\
\text { nado. }\end{array}$ & $\begin{array}{l}\text { Dh }=\left[\left(\frac{X i j}{T i}\right)\right] * 100 \\
\text { Donde xij es la cantidad de personas en el ambiente } \\
\text { i del sexo j; y Ti es el total de personas en el am- } \\
\text { biente i. }\end{array}$ & $\begin{array}{l}\text { Porcentaje de personas del sexo j dentro } \\
\text { del total de la población en el ambiente i. }\end{array}$ \\
\hline $\begin{array}{l}\text { Distribución } \\
\text { vertical }\end{array}$ & $\begin{array}{l}\text { Concentración de los individuos de } \\
\text { un mismo sexo entre los distintos } \\
\text { nombramientos, actividades, carre- } \\
\text { ras o planteles. }\end{array}$ & $\begin{array}{l}\text { Dv }=[(\mathrm{Xij} / \mathrm{Tj})]^{\star 100} \\
\text { Siendo xij la cantidad de personas en el nombra- } \\
\text { miento, ocupación, carrera o plantel i del sexo j; y Tj } \\
\text { es el total de personas del sexo j. }\end{array}$ & $\begin{array}{l}\text { Porcentaje de personas del sexo j en el } \\
\text { nombramiento, actividad, carrera o plan- } \\
\text { tel i, dentro del total de la población del } \\
\text { sexo j. }\end{array}$ \\
\hline $\begin{array}{l}\text { Índice de } \\
\text { feminidad }\end{array}$ & $\begin{array}{l}\text { Número de mujeres por cada } 100 \\
\text { hombres dentro de una población } 0 \\
\text { conjunto en estudio. }\end{array}$ & $\begin{array}{l}\text { Es el cociente entre el número de mujeres y el nú- } \\
\text { mero de hombres en una población } \\
\text { IF }=\left[\left(\frac{\mathrm{Xm}}{\mathrm{Xh}}\right)\right] * 100 \\
\text { Donde xm es la cantidad de mujeres y xh es la canti- } \\
\text { dad de hombres dentro de una población o conjunto } \\
\text { en estudio }\end{array}$ & $\begin{array}{l}\text { Número de mujeres por cada } 100 \text { hom- } \\
\text { bres. }\end{array}$ \\
\hline $\begin{array}{l}\text { Índice de } \\
\text { masculini- } \\
\text { dad }\end{array}$ & $\begin{array}{l}\text { Número de hombres por cada } 100 \\
\text { mujeres dentro de una población } 0 \\
\text { conjunto en estudio. }\end{array}$ & $\begin{array}{l}\text { Es el cociente entre el número de hombres y el nú- } \\
\text { mero de mujeres en una población } \\
\text { IF }=\left[\left(\frac{\mathrm{Xh}}{\mathrm{Xm}}\right)\right] * 100 \\
\text { Donde xm es la cantidad de mujeres y xh es la canti- } \\
\text { dad de hombres dentro de una población o conjunto } \\
\text { en estudio. }\end{array}$ & $\begin{array}{l}\text { Número de hombres por cada } 100 \text { mu- } \\
\text { jeres. }\end{array}$ \\
\hline $\begin{array}{l}\text { Índice de } \\
\text { Duncan } \\
\text { (indicador } \\
\text { de } \\
\text { segrega- } \\
\text { ción) }\end{array}$ & $\begin{array}{l}\text { Porcentaje de mujeres y de hom- } \\
\text { bres que tendrían que cambiar de } \\
\text { nombramiento, de puesto o de ca- } \\
\text { rrera para que hombres y mujeres } \\
\text { estuvieran distribuidos de manera } \\
\text { equitativa. }\end{array}$ & $\begin{aligned} \mathrm{ID}= & \frac{1}{2} \sum_{\mathrm{i}=1}^{\mathrm{i}=\mathrm{n}} \mid \frac{\text { Mujeres en la ocupación } \mathrm{i}}{\text { Empleo total femenino }}- \\
& \frac{\text { Hombres en la ocupación } \mathrm{i}}{\text { empleo total masculino }} \mid\end{aligned}$ & $\begin{array}{l}\text { Con este índice se calcula la diferencia } \\
\text { entre la proporción de individuos del gru- } \\
\text { po minoritario }(X) \text { y la proporción del res- } \\
\text { to de población. Este índice puede tomar } \\
\text { cualquier valor comprendido entre } 0 \text { y } 1 \text {, } \\
\text { y cuanto más alto sea el valor, expresa } \\
\text { mayor grado de segregación. Donde no } \\
\text { hay segregación, el índice de Duncan es } \\
\text { igual a cero. El valor } 1 \text { implica segrega- } \\
\text { ción completa; cada ocupación es com- } \\
\text { pletamente masculina o femenina. }\end{array}$ \\
\hline $\begin{array}{l}\text { Índice de } \\
\text { disimila- } \\
\text { ridad de } \\
\text { Duncan } \\
\text { (IDD) } \\
\text { por sexo }\end{array}$ & $\begin{array}{l}\text { Proporción de hombres (mujeres) } \\
\text { que deberían cambiar de ocupación } \\
\text { para mantener una proporción de } \\
\text { hombres y mujeres en la ocupación } \\
\text { igual a la que se registra a nivel del } \\
\text { total de ocupados }\end{array}$ & $\begin{array}{l}\mathrm{D}=\frac{1}{2} \sum_{\mathrm{j}=1}^{\mathrm{j}=\mathrm{n}}\left|\left(\frac{\mathrm{Fj}}{\mathrm{F}}\right)-\left(\frac{\mathrm{Mj}}{\mathrm{M}}\right)\right| * 100 \\
\text { Fj es el número de mujeres en la ocupación j, F es } \\
\text { el total de mujeres ocupadas, Mj es el número de } \\
\text { hombres en la ocupación j y M es el total de hom- } \\
\text { bres ocupados. }\end{array}$ & $\begin{array}{l}\text { Porcentaje del total de ocupados que de- } \\
\text { bería cambiar de ocupación para lograr } \\
\text { una correspondencia exacta entre la pro- } \\
\text { porción de mujeres en cada ocupación } \\
\text { y la proporción de mujeres en el total de } \\
\text { ocupados. } \\
\text { El índice de Duncan varía desde un valor } \\
\text { mínimo de } 0 \text { (cero), cuando no existe se- } \\
\text { gregación y la proporción de mujeres es } \\
\text { igual en todas las ocupaciones, hasta un } \\
\text { valor máximo de } 100 \text { cuando existen sólo } \\
\text { ocupaciones que son } 100 \% \text { masculinas } 0 \\
100 \% \text { femeninas. }\end{array}$ \\
\hline
\end{tabular}




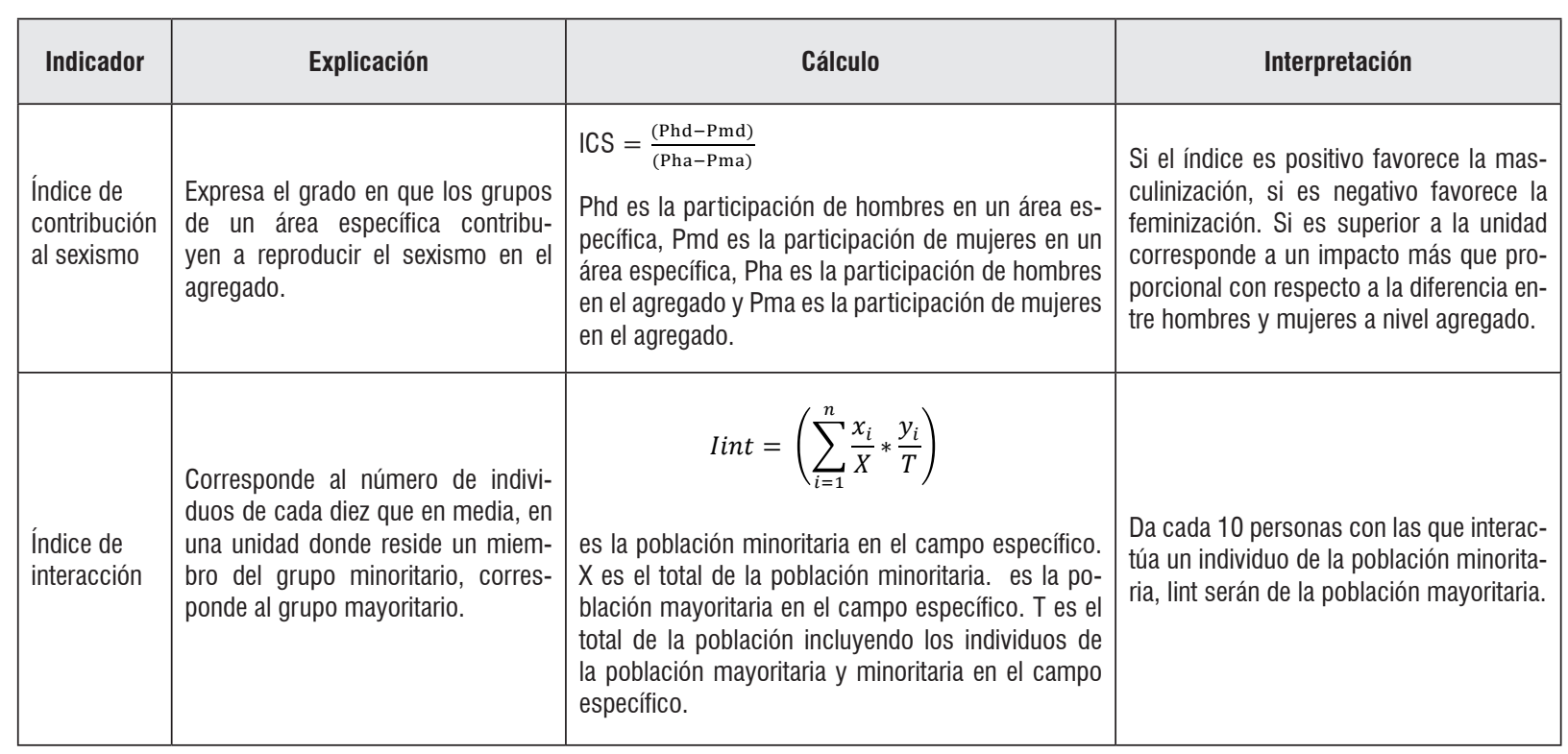

Fuente: Lis-Gutiérrez (2012). 